\title{
Externalización de procesos de negocio en tecnologías de la información y la gestión estratégica del conocimiento. Una revisión de la literatura*
}

\author{
Business process outsourcing in information technology and strategic management \\ of knowledge. A literature review
}

\author{
Acosta Prado, Julio César* \\ Hernández Reyes, Reynaldo***
}

\begin{abstract}
RESUMEN
El propósito de esta revisión de literatura es analizar si existe relación entre la externalización de procesos de negocio (EPN) en tecnologías de la información (TI) y, la gestión estratégica del conocimiento (GEC). El estudio realizado permitió conocer las principales aproximaciones que se han venido conformando, desde la década de los ochenta hasta la actualidad y que, a su vez, han marcado el enfoque investigativo en ambas áreas. Desde el punto de vista teórico, la principal contribución del estudio es la vinculación de dos marcos conceptuales (externalización de procesos de negocio y gestión estratégica del conocimiento), que apenas han sido tratados en conjunto en investigaciones precedentes sobre el tema. Desde un punto de vista práctico, los hallazgos identifican que la EPN en TI tiene efectos directos e indirectos al interior de las organizaciones, que influyen de forma positiva o negativa en la GEC para alcanzar competitividad y, por ende, éxito empresarial.
\end{abstract}

Palabras clave: Externalización de procesos de negocio, tecnologías de información, gestión estratégica del conocimiento, competitividad.

\begin{abstract}
The purpose of this literature review is to know if there's relationship between the business process outsourcing (BPO) in the area of information technology (IT) and strategic management of knowledge (SMK). The study allowed establishing the main approaches from the 80's until present times marking the investigative focus on both areas. From the theoretical point of view, the principal contribution of the study is the entailment of two conceptual frames (Business Process Outsourcing and Strategic Knowledge Management), that have barely been treated together in preceding investigations about the subject. From the practical point of view, the findings identify that BPO in IT has direct and indirect effects in the interior of organizations, that it influences of way positive and negative in the SMK to obtain competitiveness and thereby business success.
\end{abstract}

Keywords: Business process outsourcing, information technologies, strategic management of knowledge, competitiveness.

\section{INTRODUCCION}

El propósito de la revisión de la literatura es conocer si existe relación entre la externalización de procesos de negocio (EPN) en tecnologías de la información ( $\mathrm{TI}$ ) y la gestión de estratégica del conocimiento (GEC). El estudio abarca la descripción y análisis de dos modelos de gestión empresarial que desde la década de los 80 del siglo $X X$, han cobrado importancia en las decisiones estratégicas de las empresas, a fin de incrementar la competitividad y, por ende, éxito empresarial.
La EPN también conocido en inglés como Business Process Outsourcing (BPO) consiste, fundamentalmente, en la contratación con un proveedor externo de una actividad que anteriormente se realizaba en la empresa, o incluso, de nuevas actividades (Casani, et al., 1998; Greaver, 1999; Espino y Padrón, 2004). Este modelo de gestión empresarial surgió en la década de los ochenta y estuvo enfocado básicamente, en la contratación externa de los procesos relacionados con el manejo de las Tecnologías de Información (TI) (Espino y Padrón, 2004), entendiendo por éstas, la utilización de computadores para el manejo y

Artículo de revisión. Recibido diciembre 02 de 2012. Aceptado diciembre 15 de 2012.

Doctor en Dirección y Organización de Empresas. Docente Investigador, Coordinador de Investigación y Desarrollo de la Facultad de Administración de Empresas de la Universidad Externado de Colombia. Email: julioc.acosta@uexternado.edu.co

Estudiante de la Maestría en Administración de Empresas de la Universidad Externado de Colombia. Email: Reynaldo_hernandez@hotmail.com 
procesamiento de información, específicamente captura, transformación, abastecimiento, protección y recuperación de datos e información (Belogna y Walsh, 1997).

En sus inicios, que las empresas comenzaran a externalizar este tipo de procesos obedeció a que, para la época, la utilización de $\mathrm{TI}$ en los negocios apenas comenzaba a ser de uso masivo en los países industrializados, siendo escaso el personal capacitado para su manejo eficiente, por lo que se consideraba más rentable, contratar con proveedores especializados actividades del área de $\mathrm{TI}$, que incurrir en los costos de compra de equipos, sistematización de los procesos y capacitación del personal.

Sin embargo, en las últimas dos décadas del siglo $X X$ y a lo largo del siglo XXI, con el avance científico y tecnológico de la sociedad, las TI han alcanzado niveles de uso y aplicación de gran amplitud e impacto tanto en la vida cotidiana de las personas como en el manejo de las empresas. De ahí que, hoy en día, las grandes, medianas y, cada vez más, las pequeñas empresas alrededor del mundo, tienen incorporado el uso de las TI en su operación diaria.

Tal ha sido el impacto de las $\mathrm{Tl}$ en el incremento de la competitividad de las organizaciones, que actualmente la decisión de externalizar procesos en esta área, es una decisión estratégica de alto impacto para la generación de ventaja competitiva, en la medida que ya no está en juego únicamente los costos y eficiencia en las operaciones(Espino y Padrón, 2004), sino que también debe considerarse la generación y gestión de conocimientos resultado del desarrollo de nuevas habilidades y capacidades tecnológicas que permiten reconocer oportunidades de negocio y adaptación al entorno, nos referimos a la GEC (Díaz, 2006).

Por su parte, la GEC, nace a mediados de la década de los ochenta y, tiene como objetivo generar, compartir y utilizar el conocimiento tácito (saber-hacer; experiencia) y explícito (formal) existente en una organización (Nonaka y Takeuchi, 1995), como un proceso sistemático e intencionado de creación, compartición y aplicación de conocimiento crítico para el desarrollo de estrategias de negocio (Ansoff, 1980; Machlup, 1980; Nelson y Winter, 1982; Prahalad y Bettis, 1986; Wernerfelt, 1984; Dierickx y Cool, 1989; Nonaka, 1994; Nonaka y Takeuchi, 1995, Bueno, 2004). La GEC se centra en la necesidad de gestionar el conocimiento y niveles de aprendizaje en las organizaciones como mecanismo clave para el fortalecimiento de las empresas en relación con la visión de futuro que van a determinar sus planes estratégicos de desarrollo en el medio y largo plazo (Peluffo y Catalán, 2002).

Las empresas gestionan su conocimiento para crear, con base en el ya existente, uno nuevo que se traduzca en innovación, bien sea de productos y/o procesos. El fin último de todo ello es crear para la empresa destrezas, habilidades $y$, en suma, ventajas competitivas que le permitan diferenciarse y competir con éxito en el mercado, generando así resultados superiores (Nonaka y Takeuchi, 1995).

No obstante, la multidisciplinariedad inherente al estudio de la GEC y su relación con el uso eficiente de las TI, supone la existencia de diferentes perspectivas, sistemas y modelos propuestos tanto académicos como empresariales. La revisión de algunos de estos modelos en la literatura, nos permite agruparlos en tres tipos según el núcleo, los objetivos, la metodología, los participantes, etc., alrededor del cual se desarrollan, como se muestra en la tabla 1.

Como en cualquier otra área de conocimiento, las tipologías teóricas y reduccionistas arriba mencionadas, difícilmente se darán en estado puro en la realidad, más bien tienden a difuminarse y mezclarse unas con otras. De hecho, la mejor opción para desarrollar un modelo para la GEC, es basarlo en una perspectiva ecléctica que considere los aspectos fundamentales de todas ellas y las necesidades organizacionales.

No obstante, las tipologías de los modelos de la GEC mencionados guardan relación con la forma en que la EPN en TI ha ido tomando presencia en las decisiones estratégicas de las empresas. En este sentido, Accenture (2002) identifica tres tipos de EPN: Tradicional, que se realiza mediante una transferencia de la gestión o administración de un proceso o función desde el personal interno a un proveedor de servicio externo; Colaborativo, en el que se adquieren nuevas capacidades tecnológicas para la empresa cliente para mejorar en la gestión de determinados servicios y; Transformacional, que como su nombre indica busca la transformación del modo en que funciona el negocio para lograr una mejora inmediata y sostenible en el nivel de gestión empresarial.

Esta situación cobra mayor relevancia cuando la EPN es asumida como una función dinámica, bien sea de apoyo o crítica, complementaria para fortalecer las competencias esenciales y el nivel de decisión de las empresas, aun cuando el uso, aplicación y desarrollo de las TI no sea una competencia básica, como se muestra en la tabla 2.

Todo ello, permite inferir que ambos modelos de gestión tanto la EPN en TI como la GEC convergen en la generación de ventajas competitivas. Dicho esto, la pregunta de investigación es ¿Existe relación positiva o negativa entre la EPN en TI y la GEC al interior de las empresas? Es decir, hasta qué punto la EPN puede favorecer la GEC dentro de la empresa, haciendo que la gerencia se concentre en lo que compete verdaderamente en la razón de ser del negocio; y hasta dónde podría limitarla al negarle la posibilidad de desarrollar al interior habilidades y capacidades que le 
Tabla 1. Modelos de la GEC

\begin{tabular}{|c|c|c|c|c|}
\hline Autores & Núcleo & Objetivo & Metodología & Participantes \\
\hline $\begin{array}{l}\text { Sallis y Jones, 2002; } \\
\text { Wiig, 1997; Hedlund } \\
\text { y Nonaka, } 1993\end{array}$ & $\begin{array}{l}\text { Almacenamiento, ac- } \\
\text { ceso y transferencia } \\
\text { de conocimiento }\end{array}$ & $\begin{array}{l}\text { Centrados en el desarrollo } \\
\text { de metodologías, estrategias } \\
\text { y técnicas para almacenar } \\
\text { el conocimiento disponible } \\
\text { en la organización en de- } \\
\text { pósitos de fácil acceso para } \\
\text { propiciar su posterior trans- } \\
\text { ferencia entre los miembros } \\
\text { de la organización }\end{array}$ & $\begin{array}{l}\text { No suelen distinguir el cono- } \\
\text { cimiento de la información } \\
\text { y los datos. Establecen tres } \\
\text { tipos básicos de almacenes } \\
\text { de conocimiento: conoci- } \\
\text { miento externo, conocimien- } \\
\text { to interno estructurado y co- } \\
\text { nocimiento interno informal. }\end{array}$ & $\begin{array}{l}\text { Conciben el conocimien- } \\
\text { to como una entidad } \\
\text { independiente de las } \\
\text { personas que lo crean } \\
\text { y lo utilizan }\end{array}$ \\
\hline $\begin{array}{l}\text { Durán, 2004; DeTe- } \\
\text { na, 2004; Marsal y } \\
\text { Molina, 2002; No- } \\
\text { naka y Takeuchi, } \\
\text { 1995; Nonaka y } \\
\text { Konno, 1998; No- } \\
\text { naka, Reinmoeller y } \\
\text { Senno, } 1998\end{array}$ & Sociocultural & $\begin{array}{l}\text { Centrados en el desarrollo } \\
\text { de una cultura organizacio- } \\
\text { nal adecuada para el desa- } \\
\text { rrollo de procesos de GEC }\end{array}$ & $\begin{array}{l}\text { Intentan promover cambios } \\
\text { de actitudes, fomentar con- } \\
\text { fianza, estimular la creati- } \\
\text { vidad, concienciar sobre la } \\
\text { importancia y el valor del } \\
\text { conocimiento, promover la } \\
\text { comunicación y la colabo- } \\
\text { ración entre los miembros } \\
\text { de la organización }\end{array}$ & $\begin{array}{l}\text { Personas que poseen } \\
\text { el conocimiento y lo es- } \\
\text { tructuran a partir de pro- } \\
\text { cesos de socialización y } \\
\text { combinación }\end{array}$ \\
\hline $\begin{array}{l}\text { Tiwana, 2002; Tyn- } \\
\text { dale, 2002; Binney, } \\
2001\end{array}$ & Tecnológicos & $\begin{array}{l}\text { Destaca el desarrollo y la } \\
\text { utilización de sistemas (por } \\
\text { ejemplo: data warehousing, } \\
\text { intranets, sistemas expertos, } \\
\text { sistemas de información, } \\
\text { web, etc.). }\end{array}$ & $\begin{array}{l}\text { Uso de herramientas tec- } \\
\text { nológicas (por ejemplo: } \\
\text { motores de búsqueda, he- } \\
\text { rramientas multimedia y de } \\
\text { toma de decisiones) para } \\
\text { la GEC }\end{array}$ & $\begin{array}{l}\text { Personas que codifican } \\
\text { el conocimiento median- } \\
\text { te sistemas y herramien- } \\
\text { ta de TI }\end{array}$ \\
\hline
\end{tabular}

Fuente: Adaptado de Rodríguez (2006).

Tabla 2. Nivel de decisión de la EPN en TI

\begin{tabular}{|c|c|c|c|}
\hline & Nivel Táctico & & Nivel Estratégico \\
\hline & EPN Tradicional & EPN Colaborativo & EPN Transformacional \\
\hline Objetivo & $\begin{array}{l}\text { Eficiencia en cos- } \\
\text { tos y procesos de } \\
\text { negocio }\end{array}$ & $\begin{array}{l}\text { Transformación funcional o de procesos. Adquisición } \\
\text { de nuevas capacidades }\end{array}$ & $\begin{array}{l}\text { Transformación a nivel de toda la } \\
\text { organización. Centro de costos } \\
\text { convertido en centro de beneficios } \\
\text { (riesgo compartido, mayor inno- } \\
\text { vación, mejora de capacidades } \\
\text { "core", refuerzo de la posición } \\
\text { competitiva }\end{array}$ \\
\hline $\begin{array}{l}\text { Enfo- } \\
\text { que }\end{array}$ & $\begin{array}{l}\text {-Servicios estanda- } \\
\text { rizados } \\
\text {-Precio basado en } \\
\text { un porcentaje de } \\
\text { las transacciones } \\
\text {-Panorama y es- } \\
\text { cala estrecha de } \\
\text { servicios }\end{array}$ & $\begin{array}{l}\text {-Servicios flexibles } \\
\text {-Precio basado en las ganancias compartidas } \\
\text {-Servicios encaminados para alcanzar los cambios } \\
\text { deseados en el negocio }\end{array}$ & $\begin{array}{l}\text {-Servicios integrados para cam- } \\
\text { bios radicales en el negocio } \\
\text {-Basados en resultados, riesgo } \\
\text { compartidos en una estructura } \\
\text { financiera } \\
\text {-Entrega acelerada }\end{array}$ \\
\hline Función & Apoyo & Crítica & \\
\hline
\end{tabular}

Fuente: Adaptado de Accenture (2002). 
permitan crear nuevo conocimiento y desarrollar procesos continuos de innovación, que se traduzcan en competitividad $y$, por ende, el éxito empresarial.

Con base a la anterior, la revisión de la literatura recoge el desarrollo de estos dos modelos de gestión, tanto la EPN en $\mathrm{TI}$ como la GEC, a lo largo de las últimas décadas, permitiendo responder a los interrogantes que motivan la presente investigación. En este sentido; primero, se recogen los fundamentos teóricos alrededor de la EPN y de la GEC. Segundo, se presenta la metodología empleada para la revisión de la literatura. Tercero, se expone el análisis de la revisión de la literatura y; finalmente, se presentan las consideraciones finales respecto a la problemática planteada.

\section{FUNDAMENTO TEÓRICO}

La EPN es una práctica gerencial que consiste en la subcontratación con un proveedor externo a la empresa, de uno o varios procesos de negocio, entendiéndose por tales, un conjunto estructurado, medible de actividades diseñadas para producir un bien o servicio para un cliente o mercado determinado (Davenport, 1993). Los procesos de negocio, se pueden clasificar en tres categorías: 1) Procesos estratégicos, aquellos que dan orientación al negocio; 2) Procesos sustantivos, los que dan el valor al cliente, y conforman la parte principal del negocio; y, 3) Procesos de apoyo vertical $u$ horizontal que dan soporte a los procesos centrales (Hammer, 1996).

Siguiendo a Dowuona (2011), el repaso de algunas teorías prominentes que justifican la EPN en TI es presentado de la siguiente manera: Primero, desde los planteamientos de la Economía de las Organizaciones, la Teoría de Costos de Transacción (TCT). Esta teoría estudia los factores determinantes de uno y otro método alternativo de asignación de recursos, teniendo en cuenta no tanto sus costos de producción como los de transacción. Dicho esto, la transacción y el costo de producción asociado con un servicio determina la decisión de externalización (Williamson, 1975, $1979,1985)$. Este punto de vista ha sido apoyado por académicos (Shelanski y Klein, 1995; Clark et. al., 1995), que suponiendo unos costos de producción contantes, analizan las causas que originan los costos derivados de cada uno de los mecanismos de asignación de recursos.

Las fuentes de los costos de transacción se pueden agrupar en torno a dos fenómenos que, pese a estar claramente diferenciados, muestran estrechas relaciones entre sí: el comportamiento humano y el entorno en que se produce la transacción. En cuanto al comportamiento humano, la naturaleza intangible de los activos tecnológicos desafía la racionalidad limitada de los individuos (Simon, 1947) y aumenta las probabilidades de que se comporten de forma oportunista (Williamson, 1975, 1979). En este caso, la racio- nalidad limitada surge ante la dificultad para la obtención y procesamiento de la información relevante de las tecnologías objeto de transacción por parte de los decisores.

En este sentido, Lacity y Willcocks (1998) y Poppo y Zenger (2002) han sugerido que, para evitar el oportunismo, un contrato detallado y formal entre clientes y vendedores es acentuado por esta teoría. Por tanto, el origen de los comportamientos oportunistas debe buscarse en las asimetrías de información (Akerlof, 1970), producidas cuando cualquiera de las partes en una transacción posee alguna información relevante de la que las otras partes no disponen $y$, que puede aprovechar en su propio beneficio. De igual manera, Mahnke et al. (2005) argumenta que una empresa debería externalizar sus procesos de negocio cuando las dimensiones de transacción sean bajas.

El entorno en que tiene lugar la transacción es otra de las fuentes de los costos de transacción, ya que intervienen varios factores como el nivel de incertidumbre tecnológica, que origina dificultades para la identificación de las posibles contingencias y el valor real de las tecnologías en el futuro y, por ende, la redacción de contratos completos (Walker y Weber, 1984; Rindfleisch y Heide, 1997). También se identifica como factor la frecuencia de la transacción tecnológica determinante de la posibilidad de amortizar fácilmente los costos fijos derivados del establecimiento de una estructura interna para administrar la transacción. Ambos factores dificultan la identificación de: el nivel de retornos generados, el tiempo en que se van a producir esos retornos y, el área específica de aplicación (Tripsas et al., 1995). Además se deberá incluir como factor el número de contratantes, que sirve de enlace entre los costos derivados del entorno y del comportamiento oportunista. Dicho esto, a menor número de grupos contratantes los agentes podrán influir de forma directa y manifestar comportamientos oportunistas, ante la dificultar de encontrar otros alternativos para negociar (Williamson, 1975). De forma indirecta, la posesión de conocimiento tecnológico específico aumenta la probabilidad de comportamientos oportunistas, debido a la dependencia bilateral que se genera entre los agentes (Pisano, 1990; Ulset, 1996; Mang, 1998).

Sobre la base de estas consideraciones y desde un punto de vista estratégico, la TCT es un marco de análisis enriquecedor para la dirección y gestión de los intangibles tecnológicos, ya que a través del mecanismo de asignación de los activos tecnológicos que minimiza los costos de transacción, pueden deducirse los aspectos básicos que caracterizan la naturaleza de la tecnología incorporada. En otras palabras, la tecnología que posee y/o controla una empresa se sitúa entre su base de intangibles, ya que se fundamenta en información y conocimiento.

Por su parte, la Teoría de la Agencia (TA) (Jensen y Mecking, 1976; Fama y Jensen, 1983) plantea la existencia 
de la empresa como una ficción legal o nexo de contrato entre agentes cuyo fin es la consecución de objetivos que de forma autónoma no podrían realizar. La TA comparte con la TCT los supuestos básicos de racionalidad limitada y comportamiento oportunista de los individuos (Eisenhardt, 1989). Por ello, se centra en el interior de las organizaciones, principalmente en el análisis de la problemática surgida a partir de la separación entre la propiedad (accionistas) y el control (directivos) y, el modo en que los contratos firmados entre los agentes influyen en el comportamiento de las partes.

Desde la TA son diversos los problemas relacionados con el fenómeno tecnológico abordados que explican la posible disparidad de intereses y la consiguiente aparición de costos de agencia, tales como: la actitud de la dirección frente a la innovación (Hoskisson, Hitt y Hill, 1993), la relación entre diversificación y el esfuerzo tecnológico (Baysinger y Hoskisson, 1989) o el efecto de la estructura de la propiedad sobre el esfuerzo tecnológico, donde se describe de forma específica la EPN en TI (Kochhar y David, 1996), entre otros.

Para una mayor comprensión de la EPN en TI la TA centra especial atención en el riesgo que acompaña a las actividades tecnológicas y el largo período de recuperación de las inversiones. Esta situación resiente los resultados en el corto plazo y la remuneración de los directivos vinculada con ellos (Galbraith y Merrill, 1991). Por tanto, la gerencia muestra habitualmente una cierta aversión al desarrollo de actividades tecnológicas. Sin embargo, los accionistas, con una mayor capacidad para diversificar su riesgo con otras inversiones alternativas (Baysinger, Kosnik y Turk, 1991), apuestan por su ejecución debido a la evidencia empírica encontrada relativa al sentido positivo de la relación entre las inversiones en las actividades tecnológicas y la competitividad sostenida (Hoskisson, Hitt y Hill, 1993).

No obstante, el mayor problema pudiera presentarse a la hora de negociar la retribución a partir del análisis de su aportación al éxito tecnológico y a la eficacia empresarial. En este sentido, la naturaleza colectiva, compleja y específica del conocimiento tecnológico limita la capacidad de los trabajadores para negociar su retribución, permitiendo a la empresa apropiarse de los beneficios extraordinarios (Lippman y Rumelt, 2003). Por ello, en las condiciones apuntadas será éste el principal objeto de atención en el análisis de la tecnología, ya que los elevados costos derivados de una mala gestión de la citada relación de agencia pueden erosionar los resultados de las empresas. De forma análoga, los proveedores de ciertos conocimientos tecnológicos externos a la empresa contarán con un menor poder de negociación a medida que dichas tecnologías se complementen con otros conocimientos exclusivos de la empresa usuaria, que permitan mejorar su explotación frente a la utilidad comúnmente reconocida en la industria (Lippman y Rumelt, 2003), lo que puede dar lugar nuevamente a la aparición de cuasirentas.
Dicho esto, los planteamientos de la TCT y de la TA permiten reconocer, tanto el comportamiento de las empresas frente a las decisiones inherentes al desarrollo de las actividades tecnológicas al interior de las mismas o mediante su externalización, como en la alineación de la estratégica y, su impacto en la generación de ventajas competitivas. Todo ello, permite mayor la comprensión de la empresa como agente económico, ya que TCT reconoce el carácter evolutivo de los procesos de acumulación de los conocimientos tecnológicos y la necesidad de integrar factores internos y externos en el análisis estratégico y la TA analiza la evolución de los mecanismos de control, la dimensión dinámica de las organizaciones y los procesos de desarrollo tecnológico debe ser tratada con mayor detenimiento y desde una perspectiva natural que incorpore el análisis de los procesos de adaptación de la empresa a la evolución del entorno.

Sin embargo, el estudio completo de los resultados empresariales con base en la tecnología debe incluir una perspectiva dinámica que ponga el mayor énfasis posible en los procesos de desarrollo de nuevos conocimientos tecnológicos que permitan asumir los sucesivos cambios impuestos por el contexto competitivo (Bettis y Hitt, 1995; Baum y Wally, 2003). De estas observaciones, se desprende que la evolución que experimentan las empresas no es la simple respuesta a una serie de variaciones exógenas, sino que el cambio ocurre en y desde el interior de la empresa (Metcalfe, 1998). Por tanto, la actividad tecnológica se configura como un proceso endógeno a la empresa al que se le atribuyen propiedades dinámicas (Dosi, 1991).

A partir del tratamiento integrado de las perspectivas presentadas hasta el momento, se plantea el desarrollo de los enfoques estratégicos vanguardistas, que actualmente son la referencia fundamental del proceso de gestión estratégica de la empresa y que en sus desarrollos tratan de integrar las dimensiones relevantes de los enfoques anteriores.

Desde mediados de los años ochenta se desarrolla el Enfoque basado en los Recursos (EBR), que plantea el análisis de los resultados de las organizaciones a partir del control o posesión y explotación de ciertos recursos empresariales (Nelson y Winter, 1982; Rumelt, 1984, 1987; Wernerfelt, 1984, 1989; Barney, 1986, 1988, 1991; Itami y Roehl, 1987; Winter, 1987; Dierickx y Cool, 1989; Chandler, 1990; Conner, 1991; Ghemawat, 1991; Grant, 1991; Amit y Schoemaker, 1993; Peteraf, 1993;).

Este enfoque enlaza, con las primeras contribuciones al estudio de la estrategia empresarial en las que se reconoce la gran relevancia de los factores internos en la obtención de rentas superiores a través de los conceptos de competencia distintiva o heterogeneidad empresarial (Selznick, 1957; Penrose, 1959; Chandler, 1962; Ansoff, 1965; Learned et 
al., 1965; Andrews, 1971). Por tanto, las características de la industria dejan de jugar un papel exclusivo en los modelos explicativos de los resultados empresariales.

Dicho esto, autores como Wernerfelt, 1984; Prahalad y Hamel, 1990; Conner, 1991; Barney, 1991; Conner y Prahalad, 1996; Foss, 1997; Barney, 2001; Barney, Wright y Ketchen, 2001; Barney y Hesterly, 2010; Parayitam y Guru-Gharana, 2010 , visualizan las empresas como un conjunto de activos y recursos que pueden crear una ventaja competitiva si se explotan al máximo. En consecuencia, una empresa podría externalizar procesos diferentes a sus competencias básicas o capacidades únicas (Quinn, 1992; Dekkers, 2000; Fine, 2002; Wu et al., 2005; Mclvor, 2010). En este sentido, Gilley y Rasheed (2000) y Steensma y Corley (2002), argumentan que una empresa puede externalizar sus actividades de TI, si ésta no está incluida dentro de sus competencias básicas.

Por tanto, los recursos y, especialmente, las capacidades tecnológicas son los elementos con los que cuenta la empresa para neutralizar las amenazas y explotar las oportunidades que ofrece el entorno en mejores condiciones que la competencia (Conner, 1991). De una forma novedosa, Douglas y Ryman (2003) demuestran empíricamente que la habilidad de la empresa para enfrentarse al poder negociador de los clientes y a la intensidad competitiva, es a partir de su mejor dotación de capacidades tecnológicas, todo lo cual justifica su mejor posicionamiento en la industria y la obtención de mejores resultados. Sin embargo, no se hallan estudios que identifiquen resultados superiores cuando las empresas externalizan procesos de negocios orientados al desarrollo de tecnologías por no disponer de recursos suficientes internamente, lo que supondría una escasa creación y desarrollo de capacidades intensivas en conocimiento tecnológico que permitirían reconocer oportunidades del entorno competitivo o la novedad tecnológica.

La relevancia estratégica otorgada a los intangibles tecnológicos, ha derivado en el desarrollo de una corriente de pensamiento estratégico cuya separación del EBR no está suficientemente clara, nos referimos al Enfoque de la Empresa Basada en el Conocimiento (EEBC) desarrollado por Nonaka, 1994; Nonaka y Takeuchi, 1995; Bierly y Chakrabarti, 1996; Grant, 1996; Porter Liebeskind, 1996; Spender, 1996; Spender y Grant, 1996, entre otros autores. Este enfoque se preocupa no sólo de las características de los intangibles tecnológicos estratégicos, sino también por los procesos de creación, transmisión y explotación de conocimiento tecnológico en la empresa y su vinculación con la creación de valor.

A diferencia del EBR y siguiendo muy de cerca la TCT, el EEBC permite afrontar con éxito la justificación de la existencia de la empresa como un mecanismo de asignación eficiente de los intangibles tecnológicos, puesto que el me- canismo de los precios se revela insuficiente para negociar con múltiples tecnologías que son auténticas expresiones de conocimiento complejo (Machlup, 1967; Kogut y Zander, 1992, 1996; Nonaka, 1994).

Desde el EEBC, la empresa se define como una entidad de aprendizaje, almacenamiento y transmisión de conocimientos tácitos y específicos acumulados en virtud de procesos históricos (Foss, 1994, 1996) en los que la sabiduría individual y social se encarga de transformar dichos conocimientos en productos con valor económico (Demsetz, 1991; Kogut y Zander, 1992; Hall, 1993; Nonaka, 1994; Nonaka y Takeuchi, 1995; Morcillo, 1997; Grant, 1996, 2003; Spender, 1996).

Sobre la base de esas consideraciones, el EEBC enlaza con el EBR a través del desarrollo del Enfoque de las Capacidades Dinámicas (ECD), en la que, tal y como se ha presentado, el tratamiento de los procesos adquiere la mayor importancia y en la que el carácter estratégico de las capacidades tecnológicas se vincula directamente con la habilidad que otorgan a las empresas para desarrollar nuevos conocimientos continua y eficazmente responsables de la adaptación de la empresa a la evolución de la estructura competitiva (Teece, 1986, 1998; Teece y Pisano, 1994; Teece, Pisano y Shuen, 1997; Lei, Hitt y Bettis, 1996).

En coherencia con la perspectiva clásica del EBR, la dificultad para transferir e imitar los conocimientos tecnológicos tácitos y específicos justifica que los primeros trabajos identificaran exclusivamente este tipo de conocimiento como estratégico (Lippman y Rumelt, 1982; Winter, 1987; Reed y DeFillipi, 1990). Sin embargo, las aportaciones de Cohen y Levinthal (1990), apuntan a un carácter contingente con el que se reconoce que, para competir con éxito de forma sostenida, la empresa ha de renovar con agilidad y eficacia la base de tecnologías y que, en ciertas ocasiones, dichos procesos evolutivos requieren la dotación complementaria de conocimientos externos explícitos o la codificación de aquellos desarrollados internamente. Según estas aportaciones, no existiría una fórmula óptima de gestión tecnológica sino una más adecuada a la consecución de ciertos objetivos estratégicos en un contexto determinado (Pisano, 1994; Teng y Cummings, 2002).

En esta línea, Nonaka (1994) y Nonaka y Takeuchi (1995) apuntan la importancia de las interacciones de expresiones de conocimiento tácito y explícito en los procesos de creación de conocimiento tecnológico entre los distintos niveles ontológicos (individuo, grupo, organización e interorganización) así como la no sustituibilidad entre ambas dimensiones. De acuerdo a este planteamiento la EPN en $\mathrm{TI}$, genera una relación de dependencia asimétrica que podría generar riesgos y beneficios en las empresas de forma simultánea o discontinua de tiempo en la relación contractual. 
Dicho esto, cuando una empresa decide externalizar, básicamente lo que busca es enfocare en sus procesos estratégicos y sustantivos del negocio, entregando los procesos de apoyo, a proveedores especializados en dichas actividades, que le garantizan su realización con altos niveles de calidad, permitiéndole a ésta concentrarse en la razón de ser del negocio y desarrollar ventajas competitivas. Así, detrás de la externalización de un determinado proceso de negocio, la cuestión estratégica que se plantean en las organizaciones es si pueden lograr o no desarrollar una ventaja competitiva sostenible en el tiempo realizando internamente dicha actividad.

Tal cuestión se hace relevante toda vez que la experiencia ha demostrado que un uso inapropiado de la externalización o un incremento innecesario en la misma, origina, en muchas organizaciones, el inicio de un proceso de declive que puede dejarlas sin las habilidades y capacidades necesarias para competir en el mercado (Bettis et al., 1992). Por tal razón, la empresa debería ser capaz de identificar estratégicamente qué actividades puede externalizar sin que tenga que soportar riesgos innecesarios que puedan influir negativamente en su ventaja competitiva.

Así, desde el EEBC toda organización debería centrarse en aquellas actividades que constituyen sus competencias básicas y externalizar el resto teniendo en cuenta que los recursos estratégicos de la empresa deben caracterizarse por ser heterogéneos y cumplir con las propiedades de ser valiosos, escasos y difíciles de imitar o de sustituir, criterios que se relacionan con el desarrollo y la sostenibilidad de las ventajas competitivas. (Wernerfelt, 1984; Barney, 1986, 1991; Dierickx y Cool, 1989; Nonaka, 1991, 1994; Grant, 1991, 1996; Peteraf, 1993; Nenkatesan, 1992; Kugot y Zander, 1992).

Sobre la base de las consideraciones del EBR y los enfoques que se derivan de éste, tanto EEBC como ECD, para hacer uso correcto de la EPN, las actividades a externalizar deben estar en función del riesgo o la vulnerabilidad estratégica que éstas representan para la empresa. En ese sentido, si la actividad tiene una alta influencia en la ventaja competitiva de la empresa ésta demanda un alto control estratégico de la misma, por lo que se debería desarrollar internamente al suponer un gran riesgo su externalización. Sin embargo, cuando la actividad tiene una baja influencia en la ventaja competitiva, el control que se necesita es bajo y la empresa no incurriría en grandes riesgos al externalizarla dado que no va a suponer una pérdida de ventaja competitiva (Quinn, 1999; Prahalad y Hamel, 1990; Espino y Padrón, 2004).

La relación expuesta de las principales teorías y enfoques que permiten estudiar la relación propuesta entre la EPN en TI y la GEC se puede agrupar, como muestra la tabla 3 , desde los planteamientos de la economía de las organiza- ciones (TCT y TA) y los enfoques estratégicos vanguardistas (EBR, ECD y EEBC).

Sobre las base de estas consideraciones se puede inferir que cuando las empresas externalizan actividades que implican un alto riesgo para la ventaja competitiva estas podrían perder cierta flexibilidad para introducir nuevos diseños de productos o servicios, pues el nuevo diseño pasa a depender del proveedor en cuestión. También, la externalización puede erosionar el potencial de la organización para el aprendizaje organizativo particularmente en actividades necesarias para el desarrollo de aquellos negocios y capacidades básicas (Lei y Hitt, 1995), pues como señalan Cohen y Levinthal (1990) el desarrollo de actividades internas pueden ser un mecanismo eficaz para crear nuevas habilidades y capacidades básicas que se podrían perder si se confía su desarrollo a agentes externos (Rodríguez, 2004)

Espino y Padrón (2004), señalan que la externalización permite a la organización:

1. Centrarse en un conjunto de recursos limitados relativos a pocas competencias básicas en las que se pueden desarrollar las mejores capacidades.

2. Aumentar las capacidades de innovación conseguidas con el personal eficaz, la tecnología de información y el conocimiento externo.

3. Eliminar las inflexibilidades del capital fijo, la burocracia, las plantas físicas, proporcionando así unos recursos más ágiles para los clientes de la cadena de valor y de la cadena de suministros; y Expandir el propio conocimiento a través de empleo de las inversiones realizadas por fuentes externas.

De esta manera, la externalización se convierte en una decisión estratégica eficaz para cubrir las deficiencias en recursos o desfases de los resultados de las actividades en las que la empresa no es tan eficiente (Espino y Padrón, 2004).

Este planteamiento de la EPN como una cuestión estratégica, guarda relación con los planteamientos principales de la GEC, pues como ya se ha señalado la GEC se centra en la necesidad de gestionar los conocimientos y los aprendizajes organizacionales como mecanismos claves para el fortalecimiento de una organización en relación con las visiones de futuro que van a determinar sus planes estratégicos de desarrollo en el mediano y largo plazo (Peluffo y Catalán, 2002; Bueno, 2004).

La relación entre el EPN y la GEC se da en referencia a la pérdida o apropiación de destrezas transfuncionales a las 
Tabla 3. Evolución de los planteamientos estratégicos y su relación con la EPN en TI y la GEC

\begin{tabular}{|c|c|c|c|c|c|}
\hline & \multicolumn{2}{|c|}{ ECONOMÍA DE LAS ORGANIZACIONES } & \multicolumn{3}{|c|}{ PLANTEAMIENTOS ESTRATÉGICOS VANGUARDISTAS } \\
\hline & $\begin{array}{l}\text { Teoría de costos } \\
\text { de Transacción }\end{array}$ & Teoría de la Agencia & $\begin{array}{l}\text { Enfoque basado } \\
\text { en los Recursos }\end{array}$ & $\begin{array}{l}\text { Enfoque de las } \\
\text { Capacidades } \\
\text { Dinámicas }\end{array}$ & $\begin{array}{c}\text { Enfoque de la } \\
\text { Empresa basado } \\
\text { en el Conocimiento }\end{array}$ \\
\hline $\begin{array}{l}\text { Enfoque de } \\
\text { análisis }\end{array}$ & $\begin{array}{l}\text { Integrador } \\
\text { (interno/ } \\
\text { externo) } \\
\end{array}$ & $\begin{array}{l}\text { Integrador } \\
\text { (interno/externo) }\end{array}$ & $\begin{array}{l}\text { Interno } \\
\text { (estable) }\end{array}$ & $\begin{array}{l}\text { Integrador } \\
\text { (estable/ } \\
\text { dinámico) }\end{array}$ & $\begin{array}{l}\text { Integrador } \\
\text { (dinámico) }\end{array}$ \\
\hline Metodología & $\begin{array}{l}\text { Mixta } \\
\text { (cualitativa/ } \\
\text { cuantitativa) }\end{array}$ & $\begin{array}{l}\text { Mixta } \\
\text { (cualitativa/ } \\
\text { cuantitativa) }\end{array}$ & $\begin{array}{l}\text { Mixta } \\
\text { (cualitativa/ } \\
\text { cuantitativa) }\end{array}$ & $\begin{array}{l}\text { Mixta } \\
\text { (cualitativa/ } \\
\text { cuantitativa) }\end{array}$ & $\begin{array}{l}\text { Mixta } \\
\text { (cualitativa/ } \\
\text { cuantitativa) }\end{array}$ \\
\hline $\begin{array}{l}\text { Unidad de } \\
\text { análisis }\end{array}$ & Transacción & Relación de agencia & Empresa & Empresa & Empresa \\
\hline $\begin{array}{l}\text { Concepción } \\
\text { de las TI }\end{array}$ & Conocimiento & Conocimiento & Conocimiento & Conocimiento & Conocimiento \\
\hline $\begin{array}{l}\text { Utilidad en la } \\
\text { relación EPN } \\
\text { en TI y GEC }\end{array}$ & $\begin{array}{l}\text { Análisis de las } \\
\text { fuentes de acu- } \\
\text { mulación de la } \\
\text { tecnología y los } \\
\text { costos de asigna- } \\
\text { ción asociados }\end{array}$ & $\begin{array}{l}\text { Análisis de la relación } \\
\text { entre la naturaleza } \\
\text { de la tecnología y la } \\
\text { distribución de las } \\
\text { rentas generadas. }\end{array}$ & $\begin{array}{l}\text { Análisis del valor } \\
\text { estratégico de la } \\
\text { tecnología según } \\
\text { su naturaleza }\end{array}$ & $\begin{array}{l}\text { Relevancia de } \\
\text { las variables } \\
\text { del entorno y su } \\
\text { evolución en la } \\
\text { evaluación de la } \\
\text { tecnología }\end{array}$ & $\begin{array}{l}\text { Análisis de los } \\
\text { procesos de acu- } \\
\text { mulación, difusión, } \\
\text { protección y explo- } \\
\text { tación del conoci- } \\
\text { miento tecnológico }\end{array}$ \\
\hline $\begin{array}{l}\text { Principales } \\
\text { autores de } \\
\text { referencia }\end{array}$ & $\begin{array}{l}\text { Williamson, 1975, } \\
\text { 1979, 1985; } \\
\text { Shelanski y Klein, } \\
\text { 1995; Clark et. } \\
\text { al., 1995; Walker } \\
\text { y Weber, 1984; } \\
\text { Rindfleisch y Hei- } \\
\text { de, 1997; Lacity } \\
\text { y Willcocks, 1998; } \\
\text { Poppo y Zenger } \\
\text { 2002; Mahnke et } \\
\text { al. 2005 }\end{array}$ & $\begin{array}{l}\text { Jensen y Mecking, } \\
\text { 1976; Fama y Jen- } \\
\text { sen, 1983; Baysinger } \\
\text { y Hoskisson, 1989; } \\
\text { Galbraith y Merrill, } \\
\text { 1991; Baysinger, } \\
\text { Kosnik y Turk, 1991; } \\
\text { Hoskisson, Hitt y } \\
\text { Hill, 1993; Kochhar y } \\
\text { David, 1996 }\end{array}$ & $\begin{array}{l}\text { Wernerfelt, 1984; } \\
\text { Barney, 1991; } \\
\text { Conner, 1991; } \\
\text { Grant, 1991; } \\
\text { Mahoney y Pan- } \\
\text { dian, 1992; Amit } \\
\text { y Schoemaker, } \\
\text { 1993; Peteraf, } \\
\text { 1993. }\end{array}$ & $\begin{array}{l}\text { Teece, 1986, } \\
\text { 1998; Teece y } \\
\text { Pisano, 1994; } \\
\text { Teece, Pisano y } \\
\text { Shuen,1997; } \\
\text { Eisenhardt y } \\
\text { Martin, 2000. } \\
\text { Lei, Hitt y Bettis, } \\
1996\end{array}$ & $\begin{array}{l}\text { Nonaka, 1994; } \\
\text { Nonaka y Takeu- } \\
\text { chi, 1995; Bierly y } \\
\text { Chakrabarti, 1996; } \\
\text { Grant, 1996; Porter } \\
\text { Liebeskind, 1996; } \\
\text { Spender, 1996; } \\
\text { Spender y Grant, } \\
1996\end{array}$ \\
\hline
\end{tabular}

Fuente: Adaptado de García y Navas (2004).

que se exponen las empresas cuando externalizan procesos (Quinn y Hilmer, 1994), puesto que las interacciones entre las personas especializadas, bien sea por la práctica o la formación académica, de diferentes departamentos o áreas, suelen proporcionar nuevas soluciones o ideas innovadoras al corazón del negocio y sus aspectos estratégicos. Como indican Clark y Fujimoto (1990), la integración funcional de los procesos y productos enriquece la comunicación y coordinación entre las diferentes áreas. En relación a este punto, la excesiva dependencia de un proveedor por una mala selección ó abuso de las actividades a externalizar, puede dificultar la creación del conocimiento tecnológico y la transferencia del mismo a través de las diferentes funciones o departamentos de la empresa. Por consiguiente, es preciso prever y estudiar el posible impacto de la EPN en TI sobre los procesos de la GEC al interior de las empresas, entendiendo que esto repercute directamente en la capacidad de innovación y mejoramiento continuo.

\section{METODOLOGÍA}

La temática abordada en esta revisión de literatura constituyó un estudio descriptivo y analítico, desarrollado a partir de técnicas de análisis cualitativo y fuentes secundarias de información, que tuvo por objeto desarrollar un análisis de la evolución del tema escogido, su estado actual y las perspectivas de desarrollo futuro, a fin de conocer el debate teórico alrededor de la problemática planteada.

En ese sentido, el estudio se desarrolló en los siguientes momentos metodológicos:

En una primera fase se definido el problema de investigación y las variables a tratar para realizar la revisión de la literatura. Si bien se trataba de información secundaria, su carácter debía ser científico y/o académico de manera que la investigación se enmarcara dentro de los parámetros exigidos. 
Por tanto, la relación propuesta entre la EPN en TI y la GEC asumió el sesgo de selección de la búsqueda en revistas académicas asociadas a la Administración de Empresas. Desde esta perspectiva la exploración bibliográfica se realizó en tres niveles: i) revistas académicas genéricas de alto impacto en el Journal Citation Reports (JCR); ii) revistas académicas propias del campo de estudio y de alto impacto en el JCR; y, iii) revistas académicas genéricas del campo de estudio publicadas en Colombia y de alto impacto en Publindex (índice bibliográfico nacional de Colciencias).

Con base a lo anterior fueron revisados los artículos de revistas en las categorías señaladas. De la selección de artículos, se determinaron líneas centrales para el análisis de resultados del estudio realizado, organizando de una manera ágil la ruta de lectura analítica de los documentos, para determinar los hallazgos y, describir tanto las consideraciones acerca de la relación propuesta como las limitaciones.

\subsection{ANÁLISIS DE RESULTADOS}

\subsubsection{Principales hallazgos}

La dinámica investigativa afirma la proposición sobre la evolución de los conceptos a partir de los debates académicos determinados por el contexto histórico y los resultados empresariales. La revisión de literatura realizada permitió establecer la evolución de los modelo de gestión objeto de estudio y los enfoques administrativos que desde la década de los ochenta hasta la actualidad, han marcado el enfoque investigativo en ambas áreas. La tabla 4, muestra dichas tendencias:

Tabla 4. Enfoques administrativos de la EPN en TI y la GEC

\begin{tabular}{|l|l|l|l|}
\cline { 2 - 4 } \multicolumn{1}{c|}{} & Década 1980 & Década 1990 & Década 2000 \\
\hline $\begin{array}{l}\text { Nivel de } \\
\text { análisis }\end{array}$ & Organizacional & Organizacional & $\begin{array}{l}\text { Organizacional- } \\
\text { Sectorial }\end{array}$ \\
\hline $\begin{array}{l}\text { Orientación } \\
\text { Teórica }\end{array}$ & Gerencial & Gerencial & Gerencial \\
\hline $\begin{array}{l}\text { Tipo de } \\
\text { Investigación }\end{array}$ & $\begin{array}{l}\text { Empírica- } \\
\text { Conceptual }\end{array}$ & $\begin{array}{l}\text { Académica- } \\
\text { Conceptual }\end{array}$ & Empírica \\
\hline $\begin{array}{l}\text { Relación entre } \\
\text { EPN } \\
\text { y GEC }\end{array}$ & No abordada & No abordada & $\begin{array}{l}\text { Relación causal } \\
\text { débil no explícita- } \\
\text { mente determi- } \\
\text { nada }\end{array}$ \\
\hline
\end{tabular}

Fuente: Elaboración Propia

Como se muestra en la tabla 3 , el desarrollo conceptual respecto a ambos modelos de gestión empresarial, tiene su inicios en la década y en la década de los noventa se empieza a generar un soporte teórico con mayor fuerza, en especial porque para la EPN y la GEC estaban dejando de ser casos de éxito aislados para convertirse propiamente en modelos de gestión que requerían una importante robustez conceptual que los soportara. Sin embargo, la evidencia bibliográfica muestra que el desarrollo investigativo sobre EPN se ha mantenido estático en conceptos avanzando en estudios de caso y modelos empíricos que intentan establecer, tanto si los beneficios como los riesgos que está práctica gerencial reporta y, en la formulación de modelos que posibiliten a las empresas externalizar estratégicamente sin poner en riesgo su ventaja competitiva, como fue señalado al inicio con los tipos de EPN.

La bibliografía que reporta la GEC muestra que, la formulación teórica fue más fuerte en la década de los noventa, dando paso en el siglo XXI a la investigación empírica a todo nivel sobre el tema. Sin embargo, el desarrollo conceptual aun sigue en proceso ya que al hablar de conocimiento y su gestión, la investigación adopta un componente interdisciplinario, que de alguna manera imposibilita definir cualquier concepto desarrollado. Pese a todo, en los últimos años, la literatura ha intentado recoger los casos y modelos de éxito en GEC que sirvan de guía para las empresas y organizaciones, a fin de hacer posible adelantar estos modelos de gestión sin importar su razón social, tamaño y objeto de negocio.

En suma, la academia ha abordado extensamente estos temas desde dos perspectivas: una desde el desarrollo conceptual y otra más empírica desarrollada a partir de estudios de caso y proposición de modelos académicos y empresariales, susceptibles de ser aplicados.

Es claro que alrededor de ambos modelos de gestión empresarial, se ha consolidado con los años, la construcción de una comunidad académica fuerte, que se confirma en publicaciones de revistas científicas en el campo de la administración de empresas.

En este sentido, se considera robusta la producción y existencia, de conocimiento que desde la Administración, soportan ambos modelos de gestión empresarial. Gran parte de la producción bibliográfica en estos campos obedece a su carácter controversial. Así, lejos de institucionalizarse como prácticas de gestión aceptadas, tanto la EPN como la GEC son disciplinas sujetas a un extenso debate de más de tres décadas que, al parecer, aun no tiene conclusión.

En el caso de la EPN, el centro del debate está en la determinación de su conveniencia con base a las ventajas y desventajas determinadas en estudios empíricos y la evidencia existente en el interior de las empresas que la han implementado. La línea de defensa de la EPN como herramienta efectiva para obtener ventaja competitiva en las organizaciones (Hammer, 1996) ha sido ampliamente apoyada desde el enfoque basado en los recursos, enfoque del que se han desarrollado modelos estratégicos que pueden servir de guía a empresas, a fin de que ex- 
ternalicen sólo aquellas actividades que no constituyan la competencia esencial del negocio y que no comprometan las competencias básicas de la organización (Barney, 1991; Peteraf, 1993; Nenkatesan, 1992; Hammer, 1996; Quinn, 1999; Prahalad y Hamel, 1990; Espino y Padrón, 2004).

Los detractores de esta corriente afirman, con base a estudios de casos de organizaciones globalmente conocidas y con resultados de investigaciones empíricas realizadas en diferentes empresas, que la EPN no sólo constituye un riesgo para la competitividad a largo plazo sino para la seguridad de la información interna de la empresa y, para el desarrollo de procesos de innovación necesarios para liderar con éxito en el mercado mundial (Lei y Hitt, 1995; Bettis et al., 1992; Nenkatesan, 1992).

Con base en el desarrollo actual de las organizaciones y la implementación de sesiones estratégicas con el fin de generan ventaja competitiva, la generación de conocimientos internos constituye un factor clave diferenciador para el mercado. La generación de conocimientos internos no juega un papel antagónico con la EPN ya que se pueden generar sinergias con el proveedor con el fin de adquirir las capacidades y competencias generadas durante el periodo del servicio de externalización.

Pero, si de debates académicos se trata, la literatura que aborda la GEC, es extensa en todas sus formas. Pese a que en esencia los conceptos básicos que rigen este modelo de gestión empresarial son aceptados por los autores más representativos a nivel mundial en este tema (Nonaka; 1991, 1994; Nonaka y Takeuchi, 1995; Clark y Fujimoto, 1990; Grant, 1991, 1996; Peluffo y Catalán, 2002; Jaime y Blanco, 2007; Vatafu, 2010) existe un desgaste conceptual alrededor del significado de lo que es GEC desde corrientes epistemológicas, ontológicas, humanísticas y materialistas que han entrado al debate en aras de propiciar la generación de una ciencia de la GEC propiamente dicha (Bueno, 2004; Rodríguez, 2006).

Finalmente, en cuanto a la articulación de ambos conceptos, la revisión de la literatura evidencia una falta de vinculación clara entre la EPN y la GEC, tanto en investigaciones académicas como empíricas.

\subsubsection{Relación entre la EPN en TI y la GEC.}

Al abordar esta cuestión, es conveniente señalar que en la revisión de literatura adelantada se destacan tres categorías de estudio: 1) La GEC en empresas de Tecnología (Zapata, 2001; O'Dell y Grayson, 1998; Ruggles, 1998; Fiddler 2000); 2) El uso de las Tecnologías de la información y las Comunicaciones (TIC) como apoyo a los procesos de GEC y a la creación de ventajas competitivas en las organizaciones (Pérez, 1999; Pérez y Dressler, 2001; Grant, 1996; Nonaka y Teece, 2001; Knowman-Project, 2003; Andreu et al, 2004); y, 3) La importancia de la Gestión Tecnológica como apoyo fundamental para la creación y GEC (Saiz y García, 2008; Cantera, 2006),

Si bien la literatura trata de manera importante el tema de EPN, en realidad no aborda de manera específica el análisis de esta práctica dentro del área de TI en las organizaciones (Barney, 1986; Ansoff, 1980; Coyne, 1986; Lippman y Rumelt, 1982; Prahalad, y Bettis, 1986; Rumelt, 1987; Wernerfelt, 1984; Winter, 1987). En cambio, si se ha tratado la postulación de modelos de gestión como la Administración de Procesos de Negocio (APN) ó Business Process Management (BPM) como recomendación a las empresas para articular la gestión interna de la tecnología, los procesos de negocio y del conocimiento.

Lo anterior, obedece en gran parte a que la organización del siglo XXI se caracteriza por ser cada vez más flexible, dinámica y por tener incorporado dentro de todos sus procesos, o la mayoría de ellos, las TI como parte vital de su dinámica operacional. En ese sentido, hablar estrictamente de EPN dentro del área de TI, hoy en día resulta complicado y, en cierta medida simplista, dado que cada vez es más cierto que los procesos financieros, contables, de soporte técnico, de contratación, de seguridad, de calidad, de compras, gestión documental, etc., se encuentran sistematizados en las organizaciones $y$, por ende, son susceptibles de ser externalizados y de hecho lo han sido cada vez con más frecuencia en la última década.

En ese sentido, la Sociedad del Conocimiento y la Información no habla de externalizar el área de TI, sino de contratar soluciones integrales de procesos de negocio, tema distinto a externalización entendida como compra de equipos y programas informáticos manejados por un proveedor especializado para reducir costos. Así mismo, se habla de la importancia de incorporar de manera eficiente las $\mathrm{TI}$ en la estrategia corporativa de las empresas y de los riesgos de la externalización y las ventajas de la gestión tecnológica en empresas pertenecientes al sector de las $\mathrm{TI}$, tema distinto al abordado por la investigación.

En medio de este debate, el común denominador de los autores es resaltar el papel trasversal que juegan las TI para el buen desempeño de las empresas. De esa forma, las TI resultan indispensables para la creación y sostenibilidad de ventajas competitivas en las organizaciones y también para garantizar el avance de las mismas hacia el liderazgo en el mercado y el éxito empresarial. Por lo anterior, se hace necesario que exista no solo un equipamiento tecnológico en las organizaciones o una simple sistematización de procesos, sino una verdadera gestión tecnológica

Por gestión tecnológica se entiende, el proceso por el cual se ayuda a la empresa a adquirir el conocimiento necesario 
para lograr una ventaja competitiva sostenible. Para ello, es necesario que la empresa incorpore dicho proceso dentro de su estrategia corporativa y se esfuerce, a su vez por capacitar, formar y fidelizar el capital humano para que sea éste quien haga posible una verdadera GEC al interior de las empresas valiéndose de las TI como herramientas facilitadoras del proceso, entendiendo que las nuevas herramientas tecnológicas influyen sobre el comportamiento de los trabajadores en su relación con la organización y la planificación del trabajo que ha de llevarse a cabo con ellas.

En palabras de Bueno (2004), la extensión producida por las $\mathrm{TI}$ en el capital estructural de la empresa se compone, en primer lugar, de conocimientos, procesos e instrumentos conectados por internet y disponibles en el momento en que sean necesarios y, en segundo lugar, de nuevos modelos de negocio que cambian las reglas de liderazgo del mercado.

Esto último es especialmente importante, pues los resultados de la revisión de la literatura sobre las diversas investigaciones empíricas llevadas a cabo en el tema reconocen, que una de las principales ventajas de realizar, no una capitalización tecnológica sino una verdadera gestión tecnológica que incluya de manera simultánea, la adquisición de equipos, software, aplicaciones y más importante aún, que invierta en la capacitación y formación para la generación de nuevos conocimientos que derivan en la mayoría de las veces en la creación de nuevos modelos de negocio, es generar, bien sea el desarrollo de servicios en línea, el mejoramiento de servicios y procesos de la empresa, el diseño de nuevas plataformas para interactuar con los clientes creando conceptos como "hecho a su medida", entre otros y, estas a su vez, permiten diversificar no sólo el concepto de negocio sino también el significado de la marca, creando así mayores y mejores oportunidades para competir con éxito en el mercado.

En ese sentido, las TI intensifican la generación de nuevos conocimientos de las organizaciones creando profundos vínculos recíprocos de manera multidireccional, al implicar a todos cuantos participan en la red: clientes y proveedores de contexto, de contenido, de servicios comerciales y de infraestructura. Estas relaciones dinámicas remplazan al concepto de la marca como una imagen de sentido único que un vendedor define a través de la prensa u otros medios de radiodifusión y convierten las viejas reglas de la mercadotecnia en obsoletas (Rodríguez, 2006).

La relevancia de las $\mathrm{TI}$ en las organizaciones es tal que la literatura es enfática al señalar que durante el tiempo de existencia de una empresa y dependiendo de la presión al que se vea sometida por el entorno (competencia, globalización de mercados, ciclo de vida de los productos, etc.), ésta pasa por una serie de estadios que se tipifican de acuerdo con el grado de asimilación que la empresa logra de la tecnología en la cual se basa su negocio (Pérez, 1999). Según Mejía (1998) dichos estadios son:

\section{- Dependencia}

- Dependencia Relativa

- Creatividad Incipiente

- Autosuficiencia

- Excelencia

En muchas ocasiones, el estancamiento de las empresas ocurre cuando no logran asimilar o apropiar tecnología que han adquirido mediante la compra de equipos o por asistencia técnica recibida. El grado de asimilación de una tecnología específica en una empresa es sencillamente el grado de conocimiento y entendimiento que sus empleados logran acerca de ella. Esto depende del nivel de capacitación del personal, de la actitud de la gerencia hacia la aceptación de nuevas ideas de los esquemas de comunicación que se usan en la empresa, de la importancia que tiene la capacitación, etc., (Pérez, 1999).

Entre los objetivos que persigue tanto la GEC como la gestión de la tecnología, se observa concordancia en que ambas actividades buscan alcanzar las metas del negocio por medio de la obtención y gestión del conocimiento o la tecnología que la empresa requiere para ser competitiva. Ya que la tecnología es conocimiento aplicado, no es de extrañar que los principios y actividades propios de la GEC sean aplicables a la gestión de la tecnología. No obstante, en la práctica no siempre se reconoce este hecho, lo que lleva a pobres resultados y fracasos en tareas de transferencia de tecnología (Sáenz et al., 2008). Por lo anterior, a la hora de pensar en estos aspectos se deben tenerse en cuenta las siguientes diferencias que establece la literatura entre estos modelos de gestión empresarial:

Tal y como se refleja en la tabla 5, desde la concepción teórica, se afirma que la gestión de la tecnología es parte importante de la GEC y, por tanto, sus principios deben adaptarse a los de ésta última, exigiendo un cambio de paradigma que haga posible ver la gestión de la tecnología no como el proceso para lograr la mejor adquisición de maquinaria, software o implementación de estrategias o modelos administrativos, sino como el proceso por el cual se ayuda a la empresa a adquirir el conocimiento necesario para lograr una ventaja competitiva sostenible. Recalcando a su vez que los directivos dedicados a la gestión tecnológica deben ser instruidos con base al aprendizaje en todos los niveles de la organización y, poco a poco convertirse en gerentes del conocimiento (Halawi et al., 2006).

En palabras de Grant (1996), si los gerentes de las empresas enfocaran la adquisición de tecnología como un proceso de aprendizaje organizacional, serían menos propensos a menospreciar los presupuestos destinados a la capacitación 
Tabla 5. Diferencia entre GEC y Gestión de la Tecnología

\begin{tabular}{|l|l|}
\hline \multicolumn{1}{|c|}{ Gestión Estratégica del Conocimiento } & \multicolumn{1}{c|}{ Gestión de la Tecnología } \\
\hline $\begin{array}{l}\text { Los principios de la GEC tienen en cuenta las condiciones } \\
\text { que han de darse para que el conocimiento se adquiera, se } \\
\text { difunda, se utilice y se genere en la empresa, basándose en } \\
\text { el proceso de aprendizaje y la innovación. }\end{array}$ & $\begin{array}{l}\text { La gestión de tecnología, frecuentemente pasa por alto los } \\
\text { principios de la gerencia del conocimiento por creer que no } \\
\text { son necesarios o por no entender su impacto en los resultados } \\
\text { de la gestión. }\end{array}$ \\
\hline $\begin{array}{l}\text { La GEC pretende mantener y reutilizar el conocimiento adquirido } \\
\text { sobre la base de que el conocimiento no se hace obsoleto. }\end{array}$ & $\begin{array}{l}\text { La gestión de la tecnología se basa en la premisa de que la } \\
\text { tecnología se vuelve obsoleta y debe ser remplazada, con } \\
\text { lo que lecciones aprendidas en el pasado, sencillamente se } \\
\text { dejan de un lado. }\end{array}$ \\
\hline
\end{tabular}

Fuente: Elaboración propia

y entrenamiento de sus trabajadores y, en cambio recibirían mayor productividad.

Al avanzar en el análisis, una corriente especialmente importante e interesante detectada en la revisión de la literatura, es la que aborda la forma en que las $\mathrm{TI}$ impactan los negocios. De acuerdo con Pérez (1999), esto se da en tres direcciones fundamentales: a) modificando las propias estructuras del sector industrial y las reglas del juego competitivo, b) creando nuevas fuentes de ventaja competitiva y, c) permitiendo la generación de oportunidades de negocios totalmente nuevas. Sin embargo, no basta con tener el equipamiento tecnológico al interior de la empresa porque para conseguir que ésta despliegue su verdadero potencial, debe integrarse dentro de la estrategia organizacional y, más concretamente, en una estrategia para la generación y gestión eficiente del conocimiento.

Una integración exitosa de las TI, dentro de la GEC, permitiría contar con mejores herramientas para la generación de nuevo conocimiento, su transferencia y aplicación en las funciones organizacionales, productos, procesos y servicios. Finalmente, facilitarían el control de cómo todo el conocimiento organizacional es generado, transferido, usado y enriquecido

Los significativos avances en sistemas de cómputo, en los programas, en los bancos de datos y en las telecomunicaciones refuerzan, en un movimiento en espiral ascendente, la utilización de la información como factor competitivo y, por extensión, la necesidad de desarrollar en las empresas una elevada capacidad e intensidad del uso de las TI. Este último aspecto, es uno de los tres pilares que constituyen las interrelaciones estratégicas en que se sustenta la GEC. Ante este panorama, las organizaciones que pretendan alcanzar la condición de inteligentes deberán ser capaces de dominar las TI y convertirlas en una fuente de ventajas competitivas, gracias a una destacada capacidad para obtener y utilizar, de manera eficiente y efectiva, la información proveniente del mercado y del ambiente organizacional interno para combinarla con la creatividad y la capacidad de innovación de las personas (Pérez, 1999).
Se hace claro entonces, desde la revisión de la literatura, que al interior de las empresas el conocimiento difícilmente podrá ser explotado en todo su potencial sin el empleo de las TI. Entendiendo que desde el punto de vista de las organizaciones, el conocimiento se puede definir como la información que posee valor para ella; es decir, aquella información que permite generar acciones asociadas a satisfacer las demandas del mercado y apoyar las nuevas oportunidades a través de la explotación de las competencias centrales de la organización. El apoyo que pueden entregar las TI radica en instancias tecnológicas y culturales para ayudar a la dinámica del proceso de GEC las cuales pueden ser:

- Generación de conocimiento: son las herramientas y técnicas que se enfocan a la exploración y análisis de datos, clasificadas dentro del área de la inteligencia artificial.

- Facilitador de la generación de conocimiento: son las herramientas y técnicas que facilitan el libre flujo de conocimiento dentro de la organización, correspondientes al área de la Administración de la Información, comunicación, representación y Groupware.

- Mediciones de conocimiento: Son herramientas y técnicas que facilitan la visualización de los conocimientos. (Pérez, 1999).

En ese orden de ideas, la literatura reconoce de forma general un efecto positivo de las TI en la GEC, pero no profundiza en la identificación de que tecnologías concretas y en que procesos de la GEC son más favorables su aplicación (Grant, 1996; Nonaka y Teece, 2001; Knowman-Project, 2003; Andreu et al, 2004).

De la revisión de la literatura se puede inferir que las $\mathrm{TI}$ pueden lograr un doble efecto sobre la GEC. Por un lado afectan directamente a sus procesos, facilitándolos; y por otro, la afectan indirectamente al reducir los efectos negativos que determinados elementos pueden tener sobre su desarrollo. Además, con la intención de dar una visión integral al comportamiento de las TI respecto a la GEC se incorpora un elemento fundamental, el factor humano, 
que es reconocido como el actor principal dentro de los procesos de GEC (Drucker, 2000; Bueno, 2004).

En palabras de Pérez y Dressler (2007) las TI actúan como un catalizador de la GEC, al considerarlas elementos que con su utilización adecuada son capaces de hacer reaccionar al resto de factores que intervienen en la GEC, acelerar los procesos de la misma y, permitir la creación de contextos favorables para el desarrollo y expansión del conocimiento.

Además, los autores señalan que la utilización combinada de una serie de TI ejerce un efecto positivo sobre los procesos de: socialización; exteriorización; combinación e interiorización de conocimiento mencionados por Nonaka y Takeuchi (1995) en su propuesta de conversión del conocimiento. Estos suponen una reducción de las dificultades que suponen para los procesos de GEC las denominadas barreras temporales; espaciales; jerárquicas y sociales, suponen. En el mismo sentido, la capacitación de los capital humano de la organización en relación a las TI y el grado de utilización que de las mismas hagan, se relaciona de forma positiva con el desarrollo de los procesos de GEC (Pérez y Dressler, 2007).

De esta forma, la revisión de la literatura muestra cómo el estudio de las TI ha pasado del análisis de sus efectos sobre variables económico financieras a centrar las investigaciones en el análisis de su complementariedad con recursos intangibles, como el conocimiento. Y de forma simultánea, el avance técnico ha dado lugar al desarrollo de aplicaciones informáticas especiales de gestión empresarial, centradas más en el tratamiento de información y conocimiento que en la gestión de datos. Todo lo cual, lleva a la convergencia de las $\mathrm{TI}$ con la GEC, reconociendo el papel fundamental del factor humano como elemento clave en el proceso. (Pérez y Dressler, 2007). De forma que, de acuerdo a los autores estudiados, el análisis de la relación entre las TIC y la GEC no estará completo sin considerar la relación entre las $\mathrm{TI}$ y el capital humano como variable moderadora de todo el proceso (Nonaka, 1994; Nenkatesan, 1992).

Puntualizando acerca de la EPN y su relación con las $\mathrm{TI}$, siguiendo a Cantera (2006), la flexibilidad de la estrategia corporativa se caracteriza por disponer de estructuras planas y ajustadas, fabricación simplificada, en constante cambio, con tendencias a la cesión de actividades (EPN) y la virtualización del trabajo. Ello obedece a que sólo las organizaciones empresariales más flexibles son capaces de sobrevivir en entornos económicamente hostiles y dinámicos como el mercado globalizado del siglo XXI (Saiz y García, 2008).

En este proceso de supervivencia, la importancia de la EPN está en que es una estrategia que ha demostrado ser efectiva para la obtención simultánea de ventajas competitivas de primer orden o de rango superior (Investigación+Desa rrollo+innovación: $1+D+i)$ y de segundo orden o de rango inferior (menores costos salariales) mediante la reducción de costos fijos de una empresa, gracias a producción no vital o de servicios de la empresa en el exterior de la misma (Saiz, 2008). Como resultado, a nivel macro, la EPN favorece al capital humano más especializado (Egger y Egger, 2000; Keuschnigg y Ribi, 2007), divide a la cadena de valor (Feenstra y Hanson, 1999), modifica el mercado de factores productivos (Arndt, 1999; Jones y Kierzkowski, 2000; Görg y Hanley, 2008), crea flujos comerciales entre países (Saiz, 2006) y fomenta la formación de monopolios y oligopolios (Egger y Egger, 2001).

Si bien en sus inicios la EPN fue aplicada como un intento de las empresas para reducir sus costos fijos, en tanto, que flexibilizaban su estructura productiva y optimizaban el uso de los recursos. La literatura muestra que a partir del éxito conseguido en Kodak Inc., denominado académicamente como: El Efecto Kodak, tras la implantación del megacontrato firmado con IBM, Digital Equipment Corp. y Businessland para externalizar la mayor parte de sus sistemas de información, en lo que llegó a denominarse como Externalización de Tecnologías de la Información (Loh y Venkatraman, 1992; Álvarez y Díaz, 2001), llevó a una nueva concepción de la EPN, ya no como práctica sino como estrategia, tras lo cual se comenzó a utilizar como una opción reductora de costos y de creación de crecimiento en red por empresas transnacionales situadas alrededor del mundo. (Saiz y García, 2008).

Al considerar la EPN como una decisión estratégica, una de las condiciones básicas para alcanzar el éxito, es disponer de un capital humano motivado, formado y que cuente con la experiencia profesional suficiente para continuar adelante en nuevas líneas de negocio. En este proceso, juegan un papel fundamental tanto la formación especializada como el entrenamiento y las habilidades profesionales. Por ello, una de las maneras más eficaces de ayudar a aprender a las personas es a través del entrenamiento, poniendo énfasis en ayudar a la gente a aprender que en enseñar o capacitar (Saiz y García, 2008).

Esta condición propuesta en la literatura, supedita el éxito de la EPN al hecho de que la empresa cuente en su interior con gestión eficiente del capital humano altamente calificado y comprometido con la organización, que producto de un acuerdo establecido con el proveedor elegido, se traslade a dicha entidad con el fin último de abrir nuevas líneas de negocio, aprender e incorporar a sus habilidades el knowhow de éste, incrementar sus conocimientos y nivel de especialización y transmitir a la empresa dicho conocimiento adquirido, a fin de garantizar un traslado de habilidades y ventajas competitivas del proveedor a la empresa, no sólo en el papel sino en los hechos, y también para asegurar 
que en caso de finalizar el contrato, la empresa contratante de servicios de EPN no pueda quedar en clara desventaja frente a la competencia en el sector o desprovista de las herramientas, conocimientos y habilidades necesarias para sobrevivir en el mercado.

De esta forma, la externalización genera un efecto multiplicador beneficioso, tanto para la empresa como para sus partes interesadas, debido, básicamente, a tres factores: (1) La puesta en marcha de nuevos proyectos en nichos de mercado ampliados o nuevos, lo que aumenta los ingresos potenciales; (2) La reducción de costos fijos operativos, y (3) El aumento del nivel de eficiencia económica, siempre que se dé un proceso de confianza recíproca entre las empresas aliadas y una comunicación eficaz dentro de las corporaciones (Saiz y García, 2008; Sieber et. al, 2007).

Dada la mayor competencia entre corporaciones que interactúan en un entorno de globalización económica, el proceso de externalización cuyo objetivo es la reducción de costos ha generado nuevas formas de trabajo en la economía del conocimiento, así como unos mayores dividendos para los accionistas de las empresas. Buenos resultados que tienden a crear círculos virtuosos que se van retroalimentando en el tiempo (Saiz y García, 2008)

Sin embargo, desde una visión de política corporativa, la externalización en el sector de las TI tiene como objetivo satisfacer a las partes interesadas, garantizando de forma simultánea, unas posiciones de liderazgo en el tiempo que se ven reforzadas por la reducción de costos operativos y una maximización de ingresos, lo que incrementa beneficios y crea riqueza, tanto para los gerentes como los propietarios de las empresas, respectivamente. Como resultado, se maximiza el bienestar social y se incentiva el cambio social hacia la consecución de sociedades más justas y solidarias. (Saiz y García, 2008; Bustinza, 2008; Osinski, 2004).

\subsubsection{Principales Retos}

Dentro de la diferentes posibilidades que ofrecen las $\mathrm{TI}$, la literatura identifica de manera gradual importantes retos y oportunidades en estas industrias relacionadas con la GEC: desde los sistemas de información empresariales en general, pasando por su tendencia de soportar procesos transversales funcionales y no únicamente procesos o aplicaciones independientes, hasta la comprensión de los diferentes enfoques que pueden tener los sistemas de información dirigidos propiamente a la GEC (Tarazona et. al, 2009; Pereira, 2011).

La integración de soluciones de software empresariales exige identificar las aplicaciones y prioridades más relevantes y sensibles según el negocio, para hacerlas interoperables y compatibles. Paralelamente, rescatar el valor de los procesos transversales funcionales y soportar a través de soluciones TI su modelaje, ejecución, visibilidad y optimización. Esto exige identificar los procesos críticos de cada empresa y concentrar en ellos la estrategia. Del mismo modo, los Sistemas de GEC para una organización deben definirse con una estrategia coordinada, producto de la selección de las funcionalidades más convenientes para la organización (Saiz y García, 2008; Rodríguez, 2006)

Dar estructura al conocimiento de las organizaciones es un propósito que constituye en sí mismo un plan de acción: transformar el conocimiento relevante de intangible a tangible, de informal a formal, configurando repositorios de conocimiento y promoviendo su uso y enriquecimiento. No obstante, la GEC representa un proceso dinámico, que no se limita a la gestión del repositorio de conocimiento existente, sino que promueve la generación de otros nuevos conocimientos, capaces de atender las necesidades y oportunidades emergentes (Saiz y García, 2008; Bueno, 2004).

Desde el frente empírico que aborda la EPN en TI orientados a procesos de negocio y en los sistemas de GEC, la literatura identifica que tanto la industria que ofrece las diferentes soluciones, como los usuarios que las demandan, se enfrentan a trayectorias de desarrollo que aún están bajo construcción. Las soluciones aún no han logrado la madurez que garantice plena interoperabilidad, compatibilidad, flexibilidad ni funcionalidad integral. Del mismo modo, los usuarios empresariales requieren abordar estrategias muy enfocadas a sus aspectos críticos y nucleares del negocio, a sus condiciones y oportunidades particulares para sintonizar las soluciones de las $\mathrm{Tl}$ a su medida, de modo que aporten de manera efectiva y diferenciada a la estrategia global de GEC (Díaz, 2006).

Por lo anterior, se enuncia como reto importante que las empresas, sin distinción de actividad o tamaño, vean necesario el tener un conocimiento claro sobre el impacto que tienen las $\mathrm{TI}$ en las variables que determinan la competitividad de sus productos o servicios; saber muy bien si la tecnología con que cuenta la empresa proporciona alguna ventaja respecto de la competencia en esas variables donde el impacto es importante; conocer si la tecnología tiene potencial para mejorar o si ya llegó al límite; tener muy bien identificadas las competencias tecnológicas (aquellas cosas en las que la empresa es verdaderamente buena o puede serlo); saber dónde y que tanto puede y debe usarse la tecnología para hacer más competitivo el negocio, para entonces definir los proyectos que lo permitan. Definidos los proyectos, determinar los recursos necesarios para realizarlos. Si los recursos requeridos son más de los disponibles, revisar el portafolio de proyectos y ajustarlo siempre y cuando sea conocido y cómo afectará el negocio (Ortiz y Pedroza, 2006). 
Una propuesta interesante en cuanto a la forma y propósitos de la EPN consiste en pasar de los modelos de gestión de EPN ya conocidos y avanzar hacia la incursión de las empresas en la Administración de Procesos de Negocio o Business Process Management (BPM), modelo mucho más integral desde el que se busca lograr el máximo beneficio de todas las potencialidades de los procesos de negocio en las empresas y abordar iniciativas que vayan más allá de la gestión de las tareas más básicas a través del flujo del trabajo para trascender del diseño, automatización y control de procesos individuales, para trabajar procesos compartidos y visibles a lo largo de toda la organización e integrar actividades tanto de sistemas como de personas en un ambiente de optimización de procesos que conduzca a innovación (Díaz, 2006; Huang et. al, 2004).

Para lograr tales propósitos, la literatura consultada, sugiere fijar una lista de objetivos de negocio más relevantes como, identificar los procesos que influyen en estos objetivos y luego pensar en el impacto que cada proceso tiene en mejorar directamente un objetivo estratégico. Mediante esta aproximación se podrán identificar rápidamente los procesos que conducen al éxito del negocio ya que producirán a corto y a largo plazo valor tanto estratégico como financiero para la organización. Dichos procesos representan el más valioso capital de procesos -uno de los más reconocidos "activos de conocimiento"- de una empresa y, cualquier mejora en ellos representará resultados tangibles (Díaz, 2006)

Una vez implementado este modelo de gestión empresarial (EPN), la literatura recomienda que las organizaciones deben procurar permanecer enfocadas en los procesos seleccionados y convertirlos en la pieza central de la iniciativa de la administración de procesos de negocio, pues ya sea que una compañía se encuentre iniciando un proyecto de esa categoría ó solo esté aprendiendo su significado. La capacidad de aprovechar esta nueva forma de pensamientos sobre los procesos de negocio es la misma. De ese modo, el estado en que una empresa inicia este procesos solo determina si se debe extender lo que se ha logrado a la fecha o saltar la parte básica y dirigirse a las últimas innovaciones disponibles: ir más allá de la visibilidad en tiempo real del trabajo en curso, con el propósito de entender las condiciones del negocio que afectan los procesos, así como tener la capacidad de actuar ante dichas condiciones, también en tiempo real. (Díaz, 2006; Ortiz y Pedroza, 2006).

Así, la conceptualización e investigación alrededor de la EPN, abre paso a un nuevo modelo de gestión empresarial que propone integrar los procesos vitales en la organización y su gestión, dentro de la estrategia corporativa, a fin de promover unánimemente una administración óptima de procesos que soporte y facilite la GEC al interior de las empresas, teniendo siempre presente la importancia, tanto de la gestión tecnológica como de la capitalización intelectual, como factores garantes del éxito de estas estrategias.

\section{CONSIDERACIONES FINALES}

De la revisión de la literatura podemos inferir que la relación entre el EPN y la GEC se da, tanto de forma positiva como negativa, en referencia a la apropiación o pérdida de destrezas transfuncionales a las que se exponen las empresas cuando externalizan procesos en $\mathrm{TI}$, puesto que las interacciones entre las personas especializadas, bien sea por la práctica o la formación académica, de diferentes departamentos o áreas suelen proporcionar nuevas soluciones o ideas innovadoras al corazón del negocio y sus aspectos estratégicos. Como indican Clark y Fujimoto (1990), la integración funcional de los procesos y productos enriquece la comunicación y coordinación entre las diferentes áreas, ya que la excesiva dependencia de un proveedor, puede dificultar la creación del conocimiento y la transferencia del mismo a través de las diferentes funciones o departamentos de la empresa.

En cuanto a la EPN en TI la revisión de la literatura muestra un nivel de análisis estático en cuanto a conceptos avanzando en estudios de casos y modelos empíricos que intentan establecer, si son más los beneficios que los riesgos que esta práctica gerencial reporta y, la formulación de modelos que posibiliten a las empresas externalizar estratégicamente sin poner en riesgo su ventaja competitiva. Por su parte, la literatura que aborda la GEC muestra que, la formulación teórica se inicia en la década de los ochenta y se robustece en la década de los noventa, dando paso en el siglo XXI a las investigaciones empíricas y la aplicación de modelos de gestión. Sin embargo, el desarrollo conceptual aun sigue en proceso en parte porque al hablar de conocimiento y su gestión, la investigación adopta un componente interdisciplinario, que de alguna manera imposibilita dar por absoluto cualquier concepto desarrollado. Pese a todo, en los últimos años, la literatura ha intentado recoger los casos y modelos de éxito en GEC que sirvan de guía para las empresas.

La literatura analizada evidencia dos planteamientos referidos a la EPN en TI, que al parecer son antagónicos, pero convergen en la obtención de resultados superiores. Primero, la línea de defensa de la EPN en TI como herramienta efectiva para mejorar la ventaja competitiva de las organizaciones, ampliamente apoyada desde el EBC. Con base a ese enfoque se han desarrollado modelos estratégicos, a fin de que las empresas externalicen sólo aquellas actividades que no constituyan la competencia esencial o clave y, que no comprometan las competencias básicas de la organización (Hammer, 1996; Barney, 1991; Peteraf, 1993; Nenkatesan, 1992; Argyres, 2000; Quinn, 1999; Prahalad y Hamel, 1990; Espino y Padrón, 2004). Los detractores de esta corriente afirman, que la EPN en TI no 
sólo constituye un riesgo para la competitividad en el largo plazo sino para la seguridad de la información interna de la empresa y, para el desarrollo de procesos de innovación, necesarios para liderar con éxito en el mercado (Lei y Hitt, 1995; Rodríguez, 2004; Bettis et al, 1992; Nenkatesan, 1992). No obstante, estos planteamientos difícilmente se darán en estado puro en la práctica, más bien tienden a difuminarse y mezclarse unos con otros.

En cuanto a la articulación conceptual de los dos modelos de gestión abordados por la investigación, la revisión de la literatura evidencia una falta de vinculación explícita entre la EPN en TI y la GEC, tanto en investigaciones académicas como empíricas. Se destacan básicamente tres categorías de estudio: 1) La GEC en empresas de Tecnología (Zapata, 2001; O'Dell y Grayson, 1998; Ruggles, 1998; Fiddler 2000); 2) El uso de las Tecnologías de la información y las Comunicaciones (TIC) como apoyo a los procesos de GEC y a la creación de ventajas competitivas en las organizaciones (Pérez, 1999; Pérez y Dressler, 2001; Grant, 1996; Nonaka y Teece, 2001; Knowman-Project, 2003; Andreu et al,2004); y, 3) La importancia de la Gestión Tecnológica como apoyo fundamental para la creación y GEC (Saiz y García, 2008; Cantera, 2006).

Si bien la literatura trata el tema sobre la EPN, en realidad no abarca de manera específica el análisis de esta práctica dentro del área te TI. En cambio, si se ha tratado la postulación de modelos de gestión como la Administración de Procesos de Negocio (APN) ó Business Process Management (BPM) como recomendación a las empresas para articular la gestión interna tanto de la tecnología, los procesos de negocio y del conocimiento.

Tal y como se ha señalado en los enfoques estratégicos vanguardistas, al interior de las empresas, el conocimiento difícilmente podrá ser explotado en todo su potencial sin el empleo de las TI. Entendiendo que desde el punto de vista de las organizaciones, el conocimiento se puede definir como la información que posee valor para ella; es decir, aquella información que permite generar acciones asociadas a satisfacer las demandas del mercado y apoyar las nuevas oportunidades a través de la explotación de las competencias centrales de la organización. De esta forma, la literatura reconoce de forma general un efecto positivo de las TI en la GEC si bien, no profundiza en la identificación de que tecnologías concretas y en que procesos de la GEC son más favorables su aplicación (Grant, 1996; Nonaka y Teece, 2001; Knowman-Project, 2003; Andreu et al, 2004). Del análisis adelantado se obtiene que las TI pueden lograr un doble efecto sobre la GEC. Por un lado afectan directamente a sus procesos, facilitándolos; y por otro, la afectan indirectamente al reducir los efectos negativos que determinados elementos pueden tener sobre su desarrollo, como la resistencia al cambio tecnológico, au- sencia de habilidades y capacidades para asimilar y adoptar tecnologías, entre otros. Además, con la intención de dar una visión integral al comportamiento de las TI respecto a la GEC se incorpora un elemento fundamental, el factor humano, que es reconocido como el actor principal dentro de los procesos de GEC (Drucker, 2000; Bueno, 2004).

De acuerdo a la revisión de la literatura se puede inferir que el éxito o efecto positivo de la EPN en TI se debe, principalmente, al hecho de que la empresa debe contar en su interior con un capital humano altamente calificado y comprometido con la organización, que producto de un acuerdo establecido con el proveedor elegido, se traslade a dicha entidad con el fin de abrir nuevas líneas de negocio, aprender e incorporar a sus habilidades el know-how de éste, incrementar sus conocimientos y nivel de especialización y transmitir a la empresa dicho conocimiento adquirido. El propósito es garantizar un traslado de habilidades y ventajas competitivas del proveedor a la empresa. De esta forma, la externalización genera un efecto multiplicador beneficioso, tanto para la empresa como para sus partes interesadas, debido a tres factores: (1) La puesta en marcha de nuevos proyectos en nichos de mercado ampliados o nuevos, lo que aumenta los ingresos potenciales; (2) La reducción de costos fijos operativos, y (3) El aumento del nivel de eficiencia económica, siempre que se dé un proceso de confianza recíproca entre las empresas aliadas y una comunicación eficaz dentro de las organizaciones (Saiz y García, 2008; Sieber et. al, 2007).

Finalmente, desde el punto de vista teórico, la principal contribución del estudio es la vinculación de dos marcos conceptuales (EPN en TI y GEC), que apenas han sido tratados en conjunto en investigaciones precedentes sobre el tema. Desde un punto de vista práctico, los hallazgos identifican que la externalización de procesos de negocio en tecnologías de información tiene efectos directos e indirectos al interior de las organizaciones, que influyen de forma positiva o negativa en la gestión estratégica del conocimiento para lograr competitividad y, por ende, el éxito empresarial.

\section{REFERENCIAS}

Álvarez, E. y Díaz, M.C. (2001). Externalización de sistemas de información: marco teórico y evidencia empírica, Revista de ICE, 792, junio-julio, 183-200.

Akerlof, G.A. (1970). The Market for 'Lemons': Quality Uncertainty and the Market Mechanism. The Quarterly Journal of Economics, 84 (3), 488-500.

Amit, R. y Schoemaker, P.J.H. (1993). Strategic Assets and Organizational Rent, Strategic Management Journal, 14, 33-46. 
Andreu, R; Baiget, J; Almansa, A. Y Salvaj, E. (2004). Gestión del conocimiento y competitividad en la empresa española, 2003. CapGemini-IESE.

Andrews, K.R. (1971). The Concept of Corporate Strategy, Irwin, Homewood.

Ansoff, H.I. (1965). Corporate Strategy, McGraw-Hill, NewYork.

Ansoff, H. (1980) Strategic Issue Management. Strategic Management Journal (April-June), 131-148.

Arndt, S.W. (1999). Globalisation and the Open Economy, North American Journal of Economics and Finance, 8, 71-79.

Asociación Española de Empresas de Consultoría (2008) Outsourcing Palanca de crecimiento y creación de valor. AEC. Asociación Española de Empresas de Consultoría.

Barney, J.B. (1986). Strategic Factor Markets: Expectations, Luck, and Business Strategy, Management Science, 32, 1231-1241.

Barney, J.B. (1988). Returns to Bidding Firms in Mergers and Acquisitions: Reconsidering the Relatedness Hypothesis, Strategic Management Journal, 9, 71-78.

Barney, J.B. (1991). Firm resources and sustained competitive advantage. Journal of Management, 17, 99-120.

Barney, J.B. (2001). Resource-based theories of competitive advantage: a ten-year retrospective on the resource based view. Journal of Management, 27, 643-650.

Barney, J.B. y Hesterly, W.S. (2010). Strategic Management and Competitive Advantage Concepts. Upper Saddle River, $\mathrm{NJ}$ : Pearson Prentice Hall.

Barney, J.B., Wright, M. y Ketchen, D.J. (2001). The resource-based view of the firm: ten year after 1991. Journal of Management, 27 (6), 625-641.

Baum, J.R. y Wally, S. (2003). Strategic Decision Speed and Firm Performance, Strategic Management Journal, vol. 24, pp. 1107-1129.

Baysinger, B. D., Kosnik, R.H. y Turk, T.A. (1991). Effects of Board and Ownership Structure on Corporate R\&D Strategy, Academy of Management Journal, 34, 205-214.

Baysinger, B.D. y Hoskisson, R.E (1989), diversification strategy an R\&D intensity in multiproduct firms, Academy of Management Journal, 32 (2), 310-332
Belogna, G.J. y Walsh, A.M. (1997). The Accountant's Handbook of Information Technology. John Wiley and Sons. Toronto: Canada.

Bettis, R.A. y Hitt, M.A. (1995). The New Competitive Landscape. Strategic Management Journal, 16(Special Summer Issue), 7-19.

Bettis, R., Bradley, S. y Hamel, G. (1992). Outsourcing and industrial decline. Academy of Management Executive, 6 (1), 7-22.

Bierly, P. y Chakrabarti, A. (1996). Generic Knowledge Strategies in the U.S. Pharmaceutical Industry, Strategic Management Journal, 17, número especial de invierno, 123-135.

Binney D. (2001). The knowledge management spectrum - understanding the KM landscape, Journal of Knowledge Management, 5 (1), 33-42.

Bueno, E. (2004). Fundamentos Epistemológicos de Dirección del Conocimiento Organizativo: Desarrollo, medición y gestión de intangibles. Centro de Investigación sobre la Sociedad del Conocimiento y de Desarrollo e Innovación del Parque Científico de Madrid.

Bustinza, O.F. (2008). Implicaciones del outsourcing estratégico en la determinación del resultado empresarial: Gestión del conocimiento y flexibilidad como variables moderadoras. Universidad de Granada.

Cantera, J. (2006). Un modelo pragmático de una estrategia de organización a partir de un modelo de competencias, en CANTERA, J. Y GIL, F. (Coord.): Estrategia integral e integrada de gestión de personas, Madrid: Pearson

Casani, F.; Luque, M.A.; Luque, J. y Soria, P. (1998). El outsourcing y sus consecuencias en sobre los recursos humanos en el sector financiero español". Capital humano, 111, (suplemento empleo), 36-44.

Chandler, A.D. (1962). Strategy and Structure: Chapters in the History of American Industry Enterprise, MIT Press, Cambridge.

Chandler, A.D.Jr. (1990). Scale and Scope: The Dynamics of Industrial Capitalism, The Belknap Press of Harvard University Press, Cambridge.

Clark, K.B. y Fujimoto, T. 1990. The power of product integrity. Harvard Business Review. 68 (6): 107-118.

Clark, T.D., Zmud, R.W. y McGray, G.E. (1995). The outsourcing of Information services: Transforming the nature of the 
business in the Information industry, Journal of Information Technology, 8 (1), 5-13.

Cohen, W.M. y Levinthal, D.A. (1990). Absorptive Capacity: ANew Perspective on Learning and Innovation, Administrative Science Quarterly, 35, 128-152.

Conner, K.R. (1991). A Historical Comparison of ResourceBased Theory and Five Schools of Thought Within Industrial Organization Economics: Do We Have a New Theory of the Firm? Journal of Management, 17, 121-154.

Conner, R. y Prahalad, K. (1996). A Resource- Based Theory of the Firm: Knowledge versus Opportunism, Organization Science, 7 (5), 477- 501.

Coyne, K.P. (1986). Sustainable competitive advantage-What it is and what it isn't. Business Horizons. 29 (JanuaryFebruary), 54-61.

Davenport, T. H. (1993). Process innovation; reengineering work through information technology. Boston, Harvard Business School Press.

Davenport, T. y Prusak, L. (1998). Working knowledge: How organizations manage what they know. Boston: Harvard Business School Press.

Dekkers, R. (2000). Decision models for outsourcing and core competences in manufacturing. International Journal of Production Research, 38 (17), 4085-4096.

Demsetz, H. (1991). The Theory of the Firm Revisited, in O.E. Williamson y S.G. Williamson (eds.) The Nature of the Firm, Oxford University Press, Nueva York, 159-178.

De Tena, R. (2004). Modelo de GC desde una visión humanista. En Conocimiento y gestión. D. Gallego y C. Ongallo. Pearson Educación, Madrid.

Díaz, L.V. (2006). Gestión del Conocimiento y Tecnología de Información y Comunicaciones, Revista EAN. 58, 41-59.

Dierickx, I. y Cool, K. (1989). Asset Stock Accumulation and Sustainability of Competitive Advantage. Management Science, 35, 1504-1511.

Dowuona, G. (2011). Is Ghana an Emerging Destination for Business Process Outsourcing (BPO)? A Comparative Analysis between Ghana and India. Masters Dissertation. University of York. The York Management School. Working Paper No. 64.

Dosi, G. (1991). Perspectives on evolutionary theory, Science and Public Policy, 18, (6), 353-361.
Douglas, T.J. y Ryman, J.A. (2003). Understanding Competitive Advantage in the General Hospital Industry: Evaluating Strategic Competencies, Strategic Management Journal, 24, 333-347.

Drucker, P (2000), El management del siglo XXI, Barcelona, Edhasa.

Durán, M. (2004) Auditoria general d' una empresa d' alta tecnología com a procediment inicial en la implementació d'una estrategia de formació continuada: la gestió del coneixement. Tesis doctoral. En línea disponible en: http://www. tdcat.cbuc.es/TDX-0203103-184602/index.html.

Egger, H. y Egger, P. (2000). Outsourcing and Skill-specific Employment in a Small Economy: Austria and the Fall of the Iron Curtain, University of Linz Discussion Paper, 24.

Egger, H. y Egger, P. (2001). Market Concentration and International Outsourcing, The Falkinger Group Working Paper, 21, Universidad de Zürich, Suiza

Eisenhardt, K. M. (1989). Making fast strategic decisions in high-velocity environments. Academy of Management Journal, 32 (3), 543-576

Espino y Padrón (2004). ¿Qué actividades deberían externalizar las empresas? Una aproximación bajo la perspectiva de recursos y capacidades. Investigaciones Europeas de Dirección y Economía de la Empresa, 10 (1), 209-230.

Fama, E.F. y Jensen, M.C. (1983). Separation of Ownership and Control, Journal of Law and Economics, 26, 301-325.

Feenstra, R.C. y Hanson, G.H. (1999). The Impact of Outsourcing and High-technology Capital on Wages: Estimates for the United States (1979-1990), The Quarterly Journal of Economics, 114 (3), Agosto, Cambridge, Ma.: MIT Press, 907-940.

Fiddler, L. (2000). Facilitators and Impediments to the Internal Transfer of TeamEmbodied Competences in Firms Operating in Dynamic Environments. (Tesis Doctoral, Boston University). Proquest Dissertation Abstracts.

Fine, C. (2002). Rapid-response Capability in Value Chain Design. Sloan Management Review, Winter, 69-75.

Fiol, C.M. \& Lyles, M. (1985): Organizational Learning. Academy of Management Review, 10:4, 803-813.

Foss, N.J. (1994). Realism and Evolutionary Economics, Journal of Sociology and Evolutionary Systems, 17, 21-40.

Foss, N.J. (1996). Knowledge-based Approaches to the Theory of the Firm: Some Critical Comments, Organization Science, 7, 470-476. 
Foss, N.J. (1997). Resources, Firms and Strategies: A Reader in the Resource-Based Perspective, Oxford University Press, Nueva York, 306-326.

Galbraith C.S. y Merrill, G.B. (1991). The effect of compensation program and structure on SBU competitive strategy: A study of technology-intensive firms. Strategic Management Journal 12(5), 353-370.

García, F.E. y Navas, J.E. (2004). El fenómeno tecnológico y su estudio en el pensamiento estratégico. Madri+d, 23.

Ghemawat, P. (1991). Market Incumbency and Technological Inertia, Marketing Science, 10 (2), 161-171.

Gilley, K.M. y Rasheed, A. (2000). Making more by doing less: An analysis of outsourcing and its effects on firm performance. Journal of Management, 26(4), 763-790.

Görg, H. y Hanley, A. (2008). Services Outsourcing and Innovation. An Empirical Investigation, Kiel Working Papers, 1417, Abril, Kiel (Alemania): Kiel Institute for the World Economy

Grant, R.M. (1991). The Resource-Based Theory of Competitive Advantages: Implications for Strategy Formulation. California Management Review, 114-135. Los Angeles.

Grant, R.M. (1996). Toward a Knowledge-Based Theory of the Firm. Strategic Management Journal. School of Business, Georgetown University.

Grant, R.M. (2003). Strategic Planning in a Turbulent Environment: Evidence from the Oil Majors, Strategic Management Journal, 24, 491-517.

Greaver II, M. F. (1999). Strategic outsourcing. A Structured Approach to Outsourcing Decisions and Initiatives. Amacom. New York.

Halawi, L., McCarthy, R., Aronson, J. (2006). Knowledge management and the competitive strategy of the firm Emerald. The Learning Organization

Hall, R. (1993). A Framework Linking Intangible Resources and Capabilities to Sustainable Competitive Advantage, Strategic Management Journal, 14, 607-618.

Hammer, M. (1996). Beyond Reengineering: How the process-centered organization is changing our work and lives. New York, Harper Collins Publishers.

Hedlund, G. y Nonaka, I. (1993). Models of knowledge management in the West and Japan', in Lorange, P., Chakravarthy, B., Roos, J. and Van de Ven, A (eds.), Implementing
Strategic Process: Change Learning and Co-operation. Blackwell, Oxford,117-144.

Hoskisson, Hitt y Hill, (1993) managerial incentives and investment in R\&D in large multiproducts firms, Organization Science, 4 (2), 325 - 341

Huang, L., Quaddus, M., Rowe, A. y Cheng-Po, L. (2006). An investigation into the factors affecting knowledge management adoption and practice in the life insurance businessKMRP. Knowledge Management Research y Practice.

Itami, H. y Roehl, T. (1987). Mobilizing Invisible Assets, Harvard University Press, Cambridge.

Jaime, A. y Blanco, C. (2007). La gestión de conocimientos en entidades de conocimiento. El caso de los laboratorios académicos y de las empresas de base tecnológica en Europa, Pensamiento \& gestión, 22, 168-190.

Jensen, M.C. y Meckling, W.H. (1976). Theory of the Firm: Managerial Behavior, Agency Cost, and Ownership Structure", Journal of Financial Economics, 3, 305-360.

Jones, R. W. y Kierzkowski, H. (2000) Globalization and fragmentation of production. CIES Policy Discussion Paper 0010.Kaplan, R.S. y Norton, D.P. (1996). The Balanced Scorecard: Translating strategy into action, Harvard Business School Press. Boston, USA.

Knowman-Project (2003). Knowledge Management for European Small and Meddium Sized Enterprises. European Community. Online: <http://knowman.ifw.uni-bremen.de/ impressum.es.htm>.

Kochhar, R., David, P. (1996). Institutional investors and firm innovation: A test of competing hypotheses. Strategic Management Journal, 17, 73-84.

Kogut, B. y Zander, U. (1992). Knowledge of the Firm, Combinative Capabilities, and the Replication of Technology, Organization Science, 3, 383-397.

Kogut, B. y Zander, U. (1996). What Firms Do? Coordination, Identity, and Learning, Organization Science, 7, 502-518.

Keuschnigg, C. y Ribi, E. (2007). Outsourcing, Unemployment and Welfare Policy, CEPR Discussion Papers, 6605, diciembre, Londres.

Lacity, M.C. y Willcocks, L.P. (1998). An empirical investigation of information technology sourcing practices: Lessons from experience, Management Information Systems Quarterly, 22 (3), 363-408. 
Learned, E.P.; Christensen, C.R.; Andrews, K.R. y Guth, W. (1965). Business Policy: Text and Cases, Irwin, Homewood.

Lei, D. y Hitt, M.A. (1995). Strategic Restructuring and Outsourcing: The Effect of Mergers and Acquisitions and LBOs on Building Firm Skills and Capabilities. Journal of Management, 21(5), 835-859.

Lei, D.; Hitt, M.A. y Bettis, R. (1996). Dynamic Core Competences through Meta-learning and Strategic Context, Journal of Management, 22, 549-569.

Lippman, S.A. y Rumelt, R.P. (1982). Uncertain imitability: an analysis of inter firm deficiency under competition. The Bell Journal of Economics 13: 418-438.

Lippman y Rumelt (2003) Simple rent payments for the services. Strategic Management Journal.

Loh, L. y Venkatraman, N. (1992). Determinants of IT Outsourcing: a Cross-Sectional Analysis, Journal of Management Information Systems, 9 (1), 7-24.

Machlup, F. (1967). Theories of the Firm: Marginalist, Behavioral, Managerial, American Economic Review, 57, 201-220.

Machlup, F. (1980). Knowledge: Its Creation, Distribution and Economic Significance, 1, Princeton University Press, New Jersey.

Mahnke, V., Overby, M.L. y Vang, J. (2005). Strategic Outsourcing of IT Services: Theoretical Stocktaking and Empirical Challenges, Industry and Innovation, 12(2), 205-253.

Mang, P.Y. (1998). Exploiting Innovation Options: An Empirical Analysis of R\&D-intensive Firms, Journal of Economic Behavior and Organization, 35, 229-242.

Marsal, M. y Molina, J.L. (2002). La gestión del conocimiento en las organizaciones. Colección de Negocios, Empresa y Economía. Libros en red.

Mclvor, R. (2010). The influence of capability considerations on the outsourcing. International Journal of Production Research, 48 (17), 5031-5052.

Mejía, F. J. (1998). Gestión tecnológica. Dimensiones y perspectivas. Bogotá: Programa Icfes-Tecnos, Editora Guadalupe Ltda., 59-72.

Metcalfe, J.S. (1998). Evolutionary Economics and Creative Destruction, Routledge, Londres.

Morcillo, P. (1997). Dirección estratégica de la innovación y tecnología, Civitas, Madrid.
Nelson, R. y Winter, S. (1982). An Evolutionaty Theory of Economic Change. Belknap Press. Cambridge.

Nenkatesan, R. (1992). Strategic Sourcing: to make or not to make. Harvard Business Review.

Nonaka, I. (1991). The knowledge-creating company. Harvard Business Review, 69 (6), 96-104. Boston.

Nonaka, I. (1994). A Dynamic Theory of Organizational Knowledge Creation. Organization Science, 5, 14-37.

Nonaka, I. y Konno, N. (1998). The concept of Ba: building a foundation for knowledge creation. California Management Review, 40, (3), 40-54.

Nonaka, I.; Reinmoeller, P.; Senoo, D. (1998). The "Art" of Knowledge: Systems to Capitalize on Market Knowledge. European Management Journal, 16 (6), 673-684.

Nonaka, I. y Takeuchi, H. (1995). The knowledge-creating company. Oxford University Press. Oxford.

Nonaka, I y Teece, D. (2001). Managing industrial knowledge: Creation, transfer and utilization. Thousand Oaks, Sage. California.

O'Dell, C. y Grayson, C.J. (1998), If only we knew what we know: identification and transfer of internal best practice, California Management Review, 40 (3), 154-74.

Ortiz, S., y Pedroza, A. (2006) ¿Qué es la Gestión de la Innovación y la Tecnología (GINNT)? Journal of Technology Management and Innovation.

Osinski, J. (2004). Gestión del Conocimiento: por qué es crucial para el Outsourcing. ANDI.

Parayitam, S. y Guru-Gharana, K. K. (2010). Economics of Resource Based and Dynamic Capabilities View: A Contemporary Framework. Academy of Strategic Management Journal, 9(1), 83-93.

Peluffo, A, y Catalán, C. (2002). Introducción a la gestión del conocimiento y su aplicación al sector público. Instituto Latinoamericano y del Caribe de Planificación Económica y Social ILPES.

Penrose, E. T. (1959). The Theory of the Growth of the Firm. 3rd ed. Oxford University Press, Oxford

Pereira, H. (2011). Implementación de la Gestión del Conocimiento en la empresa. CEGESTI, Éxito Empresarial, Centro de Gestión de Conocimiento. 
Pérez, D. y Dressler, M. (2007). Tecnologías de la información para la gestión del conocimiento. Intangible Capital. 3, 31-59.

Pérez, G. (1999). Gestión del conocimiento en las alianzas tecnológicas, Dirección y Organización, 22, 28-36.

Peteraf, M. A. (1993). The cornerstones of competitive advantage: A resource-based view. Strategic Management Journal. 14 (March): 179-191.

Pisano, G.P. (1990). The R\&D boundaries of the firm: an empirical analysis. Administrative Science Quarterly, 35, 153-176.

Pisano, G.P. (1994). Knowledge, Integration, and the Focus of Learning: An Empirical Analysis of Process Development, Strategic Management Journal, 15, 85-100.

Poppo, L. y Zenger, T. (2002). Do Formal Contracts and Relational Governance Function as Substitutes or Complements? Strategic Management Journal, 23, 707-25.

Porter Liebeskind, J. (1996). Knowledge, Strategy, and the Theory of the Firm, Strategic Management Journal, 17, número especial de verano, 93-107.

Prahalad, C. y Bettis, R. (1986). The dominant logic: a new linkage between diversity and performance, Strategic Management Journal, 7 (6), 485-501.

Prahalad, C.K., y Hamel, G. (1990). The core competence of the corporation. Harvard Business Review.

Quinn, J.B. (1992). Intelligent Enterprise: A knowledge and service based paradigm for industry. Free Press. New York.

Quinn, J.B. (1999). Strategic outsourcing: leveranging knowledge capabilities. Sloan Managment Review. 40 (4), 9-21

Quinn, J.B. y Hilmer, F.G., (1994). Strategic outsourcing. Sloan Management Review, 35 (4), 43-55.

Rindfleisch A. y Heide J. (1997). Transaction cost analysis: past, present, and future applications. Journal of Marketing, 61 (4), 30-54.

Reed, R. y DeFillippi, R. (1990). Causal Ambiguity, Barriers to Imitation, and Sustainable Competitive Advantage, Academy of Management Review, 15, 88-102.

Rodríguez, D. (2006). Modelos para la creación y gestión del conocimiento: una aproximación teórica, Educar, 25-39.

Ruggles, R. (1998). The estate of the notion, knowledge management in practice, California Management Review, 40 (3) spring, 80-89
Rumelt, R.P. (1984). Toward a Strategic Theory of the Firm, in M. Lamb (ed.) Competitive Strategic Management, Prentice-Hall, Englewood Cliffs, pp. 556-570.

Rumelt, R.P. (1987). Theory, Strategy, and Entrepreneurship, in D. Teece (ed.) The Competitive Challenge, Ballinger Books, Cambridge, pp. 137-158.

Saiz, J.M., y García-Ochoa, M. (2008). Externalización de servicios y alianzas estratégicas en la nueva economía del conocimiento. Economía Industrial, 370, 135-141.

Saiz, J.M (2006). La ZEE de Kaliningrado. ¿Una solución de futuro?», en Flores, C. (Dir.). De la Europa del Este al estede Europa, IV Encuentro Español de Estudios sobre la Europa Oriental, Universidad de Valencia, 579-591.

Saiz, J.M. (2008): Outsourcing y creación de empleo. ¿Una estrategia de futuro?, Madrid: FIEC.

Sallis, E., y Jones, G., (2002). Knowledge Management in Education. Enhancing Learning and Education. (1st Ed.). London, United Kingdom: Kogan Page Limited.

Selznick, P. (1957). Leadership in Administration: A Sociological Interpretation, Harper and Row, Nueva York.

Shelanski, H.A. y Klein, P.G. (1995). Empirical Research in Transaction Cost Economics. Journal of Law, Economics, and Organization, 11 (2), 335-361.

Sieber, S., Valor, J. y Porta, V. (2007). La externalización de los servicios de TIC y el Business Process Outsourcing (BPO). IESE Business School. Universidad de Navarra

Simon, H. (1947). Administrative Behavior. New York, The Free Press.

Spender, J.C. (1996). Making Knowledge the Basis of a Dynamic Theory of the Firm, Strategic Management Journal, 17, número especial de invierno, 45-62.

Spender, J.C. y Grant, R.M. (1996). Knowledge and the Firm: Overview, Strategic Management Journal, 17, número especial de invierno, 27-43.

Steensma, H.K. y Corley, K.G. (2002). Organizational Context as a Moderator of Theories on Firm Boundaries for Technology Sourcing. Academy of Management Journal, 44 (4), 271-91.

Tarazona, G.M., Silva, J. y Medina, V.H. (2009). Generación de Valor en la Gestión del Conocimiento. Seventh LACCEI Latin American and Caribbean Conference for Engineering and Technology Energy and Technology for the Americas: Education, Innovation, Technology and Practice. 
Teece, D.J. (1986). Profiting from Technological Innovation, Research Policy, 15 (6), 285-305.

Teece, D.J. (1998). Capturing Value from Knowledge Assets: The New Economy, Markets for Know-How, and Intangible Assets, California Management Review, 40 (3), 55-79.

Teece; D.J. y Pisano, G. (1994). The Dynamic Capabilities of Firms: An Introduction, Industrial and Corporate Change, 3, 537-556.

Teece, D.J., Pisano, G. y Shuen, A. (1997). Dynamic Capabilities and Strategic Management, Strategic Management Journal, 18, 509-533.

Teng, B.S. y Cummings, J.L. (2002). Trade-offs in Managing Resources and Capabilities, Academy of Management Executive, 16 (2), 81-91.

Tiwana, A. (2002). The knowledge management toolkit: orchestrating IT, strategy, and knowledges platforms. Upper Sadder River, N.J.: Prentice Hall.

Tyndale, P. (2002). A taxonomy of knowledge management software tools: origins and applications, Evaluation and Program Planning, 25, 183-190.

Ulset, S. (1996). R\&D outsourcing and contractual governance: an empirical study of commercial R\&D projects. Journal of Economic Behavior and Organization 30, 63-82.

Vatafu, E.R. (2010). Knowledge Management - The Key Resource for Become Competitive. Journal of Knowledge Management, Economics and Information Technology, 1 (1), pages 7 .

Walker, G. y Weber, D. (1984). A transaction cost-approach to make-or-buy decision. Administrative Science Quarterly, 29, (3), 373-391.
Wernerfelt, B. (1984). A Resource-based View of the Firm, Strategic Management Journal, 5, 171-180.

Wernerfelt, B. (1989). From Critical Resources to Corporate Strategy, Journal of General Management, 14, 4-12.

Wiig, K.M. (1997). Knowledge Management: an introduction and perspectives. Journal of Knowledge Management, 1 (1). 6-14.

Williamson, O.E. (1975): Markets and Hierarchies, Free Press, Nueva York.

Williamson, O.E. (1979): "Transaction-Cost Economics: The Governance of Contractual Relations", Journal of Law and Economics, vol. 22, pp. 233-261.

Williamson, O.E. (1985): The Economics Institutions of Capitalism: Firms, Markets, and

Winter, S.G. (1987). Knowledge and Competence as Strategic Assets. En Teece, E. (ed.) The Competitive Challenge - Strategies for Industrial Innovation and Renewal, Ballinger, Cambridge, 159-184.

Wu, F., Li, H., Chu, L. y Sculli, D. (2005). An Outsourcing Decision Model for Sustaining Longterm Performance. International Journal of Production Research, 43 (12), 2513-2535.

Zapata, L. (2001). La Gestión del Conocimiento en Pequeñas Empresas de Tecnología de la Información: Una Investigación Exploratoria. Document de treball. Universitat Autónoma de Barcelona.

Selznick, P. (1957). Leadership in administration: A sociological interpretation. Berkeley: University of California Press. 